\title{
Properties of Individual Hippocampal Synapses Influencing NMDA-Receptor Activation by Spontaneous Neurotransmission
}

\author{
Sarah R. Metzbower, ${ }^{1}$ Yuyoung Joo, ${ }^{2}$ David R. Benavides, ${ }^{2}$ and Thomas A. Blanpied ${ }^{1}$
}

https://doi.org/10.1523/ENEURO.0419-18.2019

${ }^{1}$ Department of Physiology and Program in Neuroscience and ${ }^{2}$ Department of Neurology, University of Maryland School of Medicine, Baltimore, Maryland, 21201

\begin{abstract}
NMDA receptor (NMDAR) activation is critical for maintenance and modification of synapse strength. Specifically, NMDAR activation by spontaneous glutamate release has been shown to mediate some forms of synaptic plasticity as well as synaptic development. Interestingly, there is evidence that within individual synapses each release mode may be segregated such that postsynaptically there are distinct pools of responsive receptors. To examine potential regulators of NMDAR activation because of spontaneous glutamate release in cultured hippocampal neurons, we used GCaMP6f imaging at single synapses in concert with confocal and superresolution imaging. Using these single-spine approaches, we found that $\mathrm{Ca}^{2+}$ entry activated by spontaneous release tends to be carried by GluN2B-NMDARs. Additionally, the amount of NMDAR activation varies greatly both between synapses and within synapses, and is unrelated to spine and synapse size, but does correlate loosely with synapse distance from the soma. Despite the critical role of spontaneous activation of NMDARs in maintaining synaptic function, their activation seems to be controlled factors other than synapse size or synapse distance from the soma. It is most likely that NMDAR activation by spontaneous release influenced variability in subsynaptic receptor position, release site position, vesicle content, and channel properties. Therefore, spontaneous activation of NMDARs appears to be regulated distinctly from other receptor types, notably AMPARs, within individual synapses.
\end{abstract}

Key words: calcium imaging; GCaMP; postsynaptic density; STORM; super-resolution

\section{Significance Statement}

Understanding the synaptic mechanisms of learning and memory is critically to the field of neuroscience and for human health. A key neurotransmitter receptor involved in learning is the NMDA receptor, and exploration of its regulation is vital. Here, we optimized optical tools to allow detailed characterization of NMDA receptor activity at single synapses, along with analysis of synaptic structural features. The amount of receptor activation is independent of synapse size, but weakly dependent on synapse position within the dendritic tree. Notably, we found that NMDA receptors activated following spontaneous neurotransmitter release tend be GluN2B-containing receptors. The unique mechanisms that regulate the number and positioning of these receptors within synapses will have important consequences for control of synaptic development and signaling. 


\section{Introduction}

NMDA-type glutamate receptor (NMDAR) activation is critical for learning and memory. This is because of the unique ability of NMDARs to allow influx of $\mathrm{Ca}^{2+}$ into dendritic spines following glutamate binding and sufficient membrane depolarization (MacDermott et al., 1986; Ascher and Nowak, 1988). It is well established that NMDAR activation by action potential (AP)-dependent neurotransmitter release initiates complex signaling within the postsynaptic density (PSD), which underlies many forms for synaptic plasticity and is essential for normal synaptic function (Kennedy, 2000; Hardingham et al., 2001; Hardingham and Bading, 2003; Higley and Sabatini, 2012; Huganir and Nicoll, 2013). However, NMDAR activation by spontaneous glutamate release, although less studied, also plays a central role in synaptic function. NMDAR-mEPSCs in hippocampus are quite small, ranging from 3 to 20 pA (Prybylowski and Wenthold, 2004; Watt et al., 2004; Prybylowski et al., 2005), and they show a high degree of variability in amplitude (Bekkers and Stevens, 1989). Nevertheless, blockade of NMDARmEPSCs induces local protein synthesis-dependent synaptic plasticity (Sutton et al., 2004, 2007; Frank et al., 2006; Aoto et al., 2008), and NMDAR-mEPSCs mediate synaptic development at distal sites (Andreae and Burrone, 2015). It has been hypothesized that the amount of $\mathrm{Ca}^{2+}$ influx through NMDARs within a synapse may lead to different downstream effects (Cummings et al., 1996) and therefore is a critical factor in determining how individual synapses function. Thus, understanding how NMDAR activation due to spontaneous release events is regulated at individual synapses is essential for our understanding of how synapses are maintained and modulated.

Though spontaneous transmission clearly utilizes mechanisms that for the most part are in common with AP-evoked transmission, some evidence has accrued to suggest that it involves unique elements both presynaptically and postsynaptically. Application of open channel blockers during evoked release led to an elimination of responses because of evoked but not spontaneous release and vice versa for NMDARs, as well as AMPA receptors. This suggests that receptors activated by spontaneous release may form a synaptic pool separate

Received October 29, 2018; accepted May 12, 2019; First published May 20, 2019.

The authors declare no competing financial interests.

Author contributions: S.R.M., D.R.B., and T.A.B. designed research; S.R.M., Y.J., and D.R.B. performed research; S.R.M. analyzed data; S.R.M., D.R.B., and T.A.B. wrote the paper.

This work was supported by F31-MH105105 and T32-GM008181 to S.R.M., R01-MH080046 and NS090644 to T.A.B. We thank Sai Sachin Divakaruni, Aaron Levy, and other members of the Blanpied Laboratory for helpful discussion, and Minerva Contreras for invaluable technical assistance.

Correspondence should be addressed to Thomas A. Blanpied at Tblanpied@som.umaryland.edu.

https://doi.org/10.1523/ENEURO.0419-18.2019

Copyright (C) 2019 Metzbower et al.

This is an open-access article distributed under the terms of the Creative Commons Attribution 4.0 International license, which permits unrestricted use, distribution and reproduction in any medium provided that the original work is properly attributed. from receptors activated by evoked release within a single synapse (Atasoy et al., 2008; Sara et al., 2011). Additionally, there is some evidence that glutamate vesicles that fuse spontaneously are drawn from a specific subset of the total vesicle pool (Sara et al., 2005; Fredj and Burrone, 2009; Ramirez and Kavalali, 2011; Andreae et al., 2012), though this is still a subject of some debate (Prange and Murphy, 1999; Groemer and Klingauf, 2007; Hua et al., 2010; Wilhelm et al., 2010). However, that spontaneous activation drives signaling pathways through NMDAR activation at individual synapses that are unique from those induced by evoked release (Sutton et al., 2007; Autry et al., 2011), supports the notion that a portion of synaptic NMDARs are specifically activated by spontaneously released glutamate. Together, this highlights the need to understand the factors at individual synapses that control the type and numbers of NMDARs activated by spontaneous release.

One such parameter could be NMDAR subunit composition, which is a major factor that influences both receptor biophysical properties and downstream signaling pathways (Paoletti et al., 2013; Shipton and Paulsen, 2014). NMDARs are typically assembled from two GluN1 subunits and two GluN2 subunits that either are two GluN2A (GluN2A-NMDARs), two GluN2B (GluN2BNMDARs), or triheteromers with one of each type of GluN2 subunit (GluN2A/B-NMDARs; Rauner and Köhr, 2011; Tovar et al., 2013; Hansen et al., 2014). These receptor types have biophysical differences in glutamate affinity, channel open time, and decay kinetics that lead to differences in $\mathrm{Ca}^{2+}$ influx magnitude and kinetics (Santucci and Raghavachari, 2008). In addition, they engage in different protein-protein interactions within the PSD to gate unique downstream signaling pathways (Paoletti et al., 2013; Shipton and Paulsen, 2014). Thus, which NMDAR subunits contribute to NMDAR activation by spontaneous release may underlie differences in receptor activation and subsequent functional effects.

For AMPA receptors (AMPARs), there is a strong relationship between the amount of receptor activation and the size of the spine (Matsuzaki et al., 2001). This suggests that synapse structure may be an additional critical factor in modulating NMDAR activation by spontaneous release. However, neither the number of NMDARs present (Kharazia and Weinberg, 1999; Takumi et al., 1999; Shinohara et al., 2008; Chen et al., 2015), nor the amount of NMDAR activation by glutamate uncaging (Sobczyk et al., 2005) or evoked release (Nimchinsky et al., 2004), was correlated with synapse size. Nevertheless, because NMDAR activation due to spontaneous release may diverge from the total synaptic NMDAR pool, we asked whether spontaneous NMDAR activation is sensitive to synaptic spine or synapse size.

Here we combined single-synapse $\mathrm{Ca}^{2+}$ imaging with confocal and super-resolution microscopy to explore the relationship between NMDAR activation due to spontaneous single vesicle exocytosis and morphologic features of individual synapses. We find that NMDAR activation by spontaneous release is mediated primarily through GluN2B-NMDARs. The amount of $\mathrm{Ca}^{2+}$ influx was not 
correlated with spine or synapse size, and only weakly correlated with synapse position on the neuron. However, the magnitude of activation was highly variable at individual synapses. Thus, the high degree of variability of spontaneous NMDAR activation is most likely dominated by stochastic channel fluctuations and by nanoscale intrinsic properties of the synapse including receptor position, release site position, and the glutamate concentration profile following each release event.

\section{Materials and Methods}

\section{Cell culture and GCaMP6f expression}

Dissociated hippocampal cultures were prepared from E18 rats of both sex and plated on glass coverslips coated with poly-L-lysine. A subset of cells was infected with pAAV.CAG.GCaMP6f.WPRE.SV40 (Penn Vector Core) at 0 DIV and plated along with uninfected cells so that on each coverslip there is a mix of infected and uninfected cells. This allowed us to vary the ratio of infected cells to uninfected cells plated based on the requirement of the experiment. For most experiments $10,000-15,000$ infected cells were plated with 40,000 uninfected cells to have a total density $\sim 50,000 /$ well. For correlative $\mathrm{Ca}^{2+}$ imaging and super-resolution experiments we used 1000 infected cells plated with 30,000 uninfected cells per well. Transfections were performed using Lipofectamine at 18 DIV with either GCaMP6f or tdTomato, and constructs were allowed to express for 3 $\mathrm{d}$, followed by imaging at 21 DIV. All animal procedures were performed in accordance with the University of Maryland Baltimore animal care and use committee's regulations.

\section{$\mathrm{Ca}^{2+}$ imaging}

$\mathrm{Ca}^{2+}$ imaging was performed on three very similar spinning disk confocal systems. The first was a CSU-22 confocal (Yokagawa) with a Zyla 4.2 sCMOS camera (Andor) mounted on the side port of an Olympus IX-81 inverted microscope, using a $60 \times / 1.42$ oil-immersion objective. The second system used the same camera, microscope, and objective as above but the CSU-22 confocal was replaced with a Dragonfly multimodal system (Andor). All time-lapses were acquired at $20 \mathrm{~Hz}$ controlled by IQ3 software (Andor). Following time-lapse acquisition, a $z$-stack of the field was acquired using 50-200 ms frames and $0.5 \mu \mathrm{m}$ steps between planes. Coverslips were imaged in ACSF containing $0 \mathrm{~mm} \mathrm{Mg}{ }^{2+}, 139 \mathrm{~mm} \mathrm{NaCl}, 2.5$ $\mathrm{mM} \mathrm{KCl}, 1.5 \mathrm{~mm} \mathrm{CaCl}_{2}, 10 \mathrm{~mm}$ glucose, and $10 \mathrm{~mm}$ HEPES, pH adjusted to 7.4 with $\mathrm{NaOH}, 1 \mu \mathrm{M}$ TTX (Enzo), $10 \mu \mathrm{M}$ DNQX (Sigma-Aldrich), $20 \mu \mathrm{M}$ ryanodine (Tocris Bioscience), $1 \mu \mathrm{M}$ thapsigargin (Sigma-Aldrich), and $5 \mu \mathrm{M}$ nifedipine (Sigma-Aldrich). All experiments were performed using an objective heater to maintain bath temperature near $37^{\circ} \mathrm{C}$. To maintain the plane of focus, autofocus was performed every minute using an Olympus ZDC2. For experiments with a treatment (adding blockers, ifenprodil, raising $\mathrm{Ca}^{2+}$, and raising $\mathrm{Mg}^{2+}$ ), baseline imaging of 4-6 min was followed by application of either drugs or vehicle (ACSF or DMSO, depending on drug) and then by an additional 4-6 min of imaging. The third spinning disk confocal system was a Nikon W1 equipped with a $40 \times / 1.3$ oil-immersion objective and Hamamatsu Flash 4.2 camera, mounted on a Ti2 microscope. This was used for imaging the larger dendritic trees in Figure 4, and images were acquired using otherwise the same parameters and conditions as above. For experiments in Extended Data Figure 2-1, experiments using transfected cells, as well as D-serine experiments were performed on a Nikon Ti2 equipped for wide-field fluorescence imaging using a $40 \times / 1.3$ oil-immersion objective and a Zyla 4.2 sCMOS camera. Autofocus was maintained continuously, and acquisition parameters were otherwise the same as above.

\section{$\mathrm{Ca}^{2+}$ imaging analysis}

To analyze the $\mathrm{Ca}^{2+}$ imaging data, averages of the first 50-100 frames were generated either in MATLAB or MetaMorph. On each averaged image, a circular regionof-interest (ROI) was drawn around every single spine that was in focus, distinct from the dendrite, and unobstructed, regardless of activity level, as well as a background ROI. Mean intensity within each region was measured for every frame using custom MATLAB scripts. Background subtraction was done by subtracting the average intensity of the background $\mathrm{ROI}$ from the average intensity of each spine ROI per frame. To calculate $\Delta F / F$, $F_{\text {baseline }}$ was determined for each spine ROI every minute by averaging fluorescence intensity every 10 frames, and within every minute interval of imaging finding the lowest positive value. Then for each frame ( $\left.F_{\text {frame }}-F_{\text {baseline }}\right) /$ $F_{\text {baseline }}$ was calculated. To detect and measure peaks, the $\Delta \mathrm{F} / \mathrm{F}$ values were then fed into Clampex (Molecular Devices) where miniature spontaneous $\mathrm{Ca}^{2+}$ transients (mSCaTs) were detected using a template search that identified peaks based on an average shape profile. For measurements normalized to baseline, only spines that had at least three mSCaTs at baseline were included, typically $\sim 23-55 \%$ of all spines imaged. While for measurements that are not normalized, all spines are included for frequency data and spines with at least one mSCaT were used for amplitude data.

\section{Spine and cell morphologic analysis}

Spine area was measured using maximum projections of the post- $\mathrm{Ca}^{2+}$ imaging GCaMP6f $z$-stacks in MetaMorph. Within each spine ROI, an intensity-based threshold was used to calculate area. To measure synapse distance from the soma and branch depth, GCaMP6f $z$-stacks were imported into Imaris (Bitplane) for semiautomatic dendrite and spine detection. Spines identified in Imaris were matched to $\mathrm{Ca}^{2+}$ imaged spines using custom MATLAB scripts.

\section{Immunocytochemistry}

For immunocytochemistry, cultured hippocampal neurons were fixed in $4 \%$ paraformaldehyde (PFA) and $4 \%$ sucrose directly following $\mathrm{Ca}^{2+}$ imaging for $20 \mathrm{~min}$ at room temperature (RT). Following fixation, coverslips were washed with PBS + glycine and permeabilized in PBS $+0.3 \%$ Triton X-100 for 20 min at RT. Next, cells were incubated in blocking buffer containing $10 \%$ donkey 
serum (DS; Sigma-Aldrich) and $0.2 \%$ Triton $\mathrm{X}-100$ for $1 \mathrm{~h}$ at room temperature. For labeling of Shank, cells were incubated in 1:200 anti-Shank primary (NeuroMab; RRID: AB_10672418), 5\% DS and $0.1 \%$ Triton $\mathrm{X}-100$ for $3 \mathrm{~h}$ at RT. Followed by incubation with 1:200 AlexaFluor 647conjugated goat anti-mouse secondary antibody (Jackson Laboratories) for $1 \mathrm{~h}$ at RT. Finally, cells were postfixed in $4 \%$ PFA and $4 \%$ sucrose for $10 \mathrm{~min}$.

\section{Super-resolution imaging and analysis}

dSTORM imaging was performed on the Olympus IX-81 inverted microscope, using a 60×/1.42 oil-immersion objective along with Dragonfly multimodal system (Andor) and an iXon + 897 EM-CCD camera (Andor). Cells that had undergone $\mathrm{Ca}^{2+}$ imaging were first located by eye in PBS, which was then replaced with STORM imaging buffer containing $50 \mathrm{~mm}$ Tris, $10 \mathrm{~mm} \mathrm{NaCl}, 10 \%$ glucose, 0.5 $\mathrm{mg} / \mathrm{ml}$ glucose oxidase (Sigma-Aldrich), $40 \mu \mathrm{g} / \mathrm{ml}$ catalase (Sigma-Aldrich), and $0.1 \mathrm{M}$ cysteamine (SigmaAldrich) before imaging. Acquisition was performed at 20 $\mathrm{Hz}$, for a total of 70,000 frames while autofocusing every 1000 frames. Imaging was conducted in wide-field mode using the Dragonfly's beam expander set to a power density of 4 to concentrate the excitation beam.

All dSTORM analysis was done using custom MATLAB (MathWorks) scripts that fit peaks with an elliptical 2D Gaussian function to a $5 \times 5$ pixel array. The fitted peaks were used to determine $x$ and $y$ coordinates of the molecules. Molecules with a localization precision $<20$ were used for analysis. PSD detection was performed using custom MATLAB scripts. Briefly, following localization detection and drift correction, the image was re-rendered with $14.75 \mathrm{~nm}$ pixels, and clusters of localizations exceeding the density cutoff of 1 localization per $217.6 \mathrm{~nm}^{2}$ were identified. Clusters with areas $<0.02 \mu \mathrm{m}^{2}$, the bottom of the range reported for synapses imaged with super-resolution (MacGillavry et al., 2013), were rejected. Spine $\mathrm{Ca}^{2+}$ data were matched to individual PSDs by overlaying super resolved PSDs on the GCaMP6f $z$-stack and manually matching spines between the post-STORM $z$-stack and the $\mathrm{Ca}^{2+}$ imaging time-lapse.

\section{Immunoblotting}

Hippocampal neuron cultures were prepared as described, infected at 0 DIV with AAV vectors, and plated at density of $\sim 100,000$ cells per well in 12-well plate. Neurons were matured as described and at 12 or 19 DIV, neuronal cell homogenates were prepared in preheated buffer containing 1\% SDS, $50 \mathrm{~mm} \mathrm{NaF}, 1 \mathrm{~mm}$ sodium orthovanadate, and Phosphatase Inhibitor Cocktail I (Sigma-Aldrich) and Phosphatase Inhibitor Cocktail III (Sigma-Aldrich) supplemented with protease inhibitors (Roche cOmplete, EDTA-free). Homogenates were collected into microcentrifuge tubes, sonicated using manual ultrasonication probe, and heated at $65^{\circ} \mathrm{C}$ for $10 \mathrm{~min}$. Protein concentration was determined by BCA assay (Pierce) and equal amounts of total protein $(10 \mu \mathrm{g})$ from each sample were resolved by SDS-PAGE and transferred to PVDF membranes for detection by near-infrared fluorescence. Total protein stain was performed on all membranes (REVERT, LI-COR Biosciences). Immunoblot images were obtained on Odyssey Imaging System and quantitated using Image Studio 4.0 software (LI-COR Biosciences). NMDAR subunit protein levels were normalized against total protein signal for each lane. Quantitative plots were constructed using normalized mean values from six samples collected from each condition on each maturation day from a single culture. Data represent mean values \pm SEM. Statistical analyses were conducted on quantitated values using GraphPad software.

\section{Antibodies}

Membranes were blotted with primary antibodies including rabbit polyclonal anti-GluN1 C-terminus (1:2000; Sigma-Aldrich, catalog \#G8913; RRID:AB_259978), rabbit polyclonal anti-GluN2A N-terminus (1:500; JH6097 gift from R. Huganir, Johns Hopkins University), mouse monoclonal anti-GluN2B C-terminus (1:2000; Millipore, catalog \#05-920; RRID:AB_417391), and mouse monoclonal anti- $\alpha$ Tubulin (1:2000; Sigma-Aldrich, catalog \#T6074; RRID:AB_477582). Near-infrared-conjugated secondary antibodies were used to detect signal for GluN1 or GluN2A: donkey anti-rabbit 680RD (1:10 000; LI-COR Biosciences, catalog \#926-68073; RRID: AB_10954442), GluN2B: donkey anti-mouse 800CW (1: 10,000; LI-COR Biosciences, catalog \#926-32212; RRID: AB_621847), and $\alpha$-tubulin: goat anti-mouse 680RD (1: 10,000; LI-COR Biosciences, catalog \#926-68070; RRID: AB_10956588).

\section{Experimental design and statistical analysis}

Unless otherwise stated, the number of spines was >200; however, treatment with ifenprodil or $\mathrm{Mg}^{2+}$ reduced event frequency so that there were fewer spines to use for mSCaT amplitude measurements post-treatment and unless otherwise stated these came from at least three separate culture preparations. Statistical analysis was performed using GraphPad Prism. Data are shown as mean \pm SEM. Kruskal-Wallis followed by Dunn's test for multiple comparisons were used for experiments that had more than two groups; otherwise Student's $t$ test was used to compare means at $p<0.05$. For experiments comparing the effect of a treatment between groups, post-treatment parameters were normalized to each spine's own baseline to assess the impact of the treatment. For correlations, Pearson's correlation coefficients were used to assess the strength of the relationship. When noted, data were binned into eight bins so that pattern in the data could be observed more clearly; however, no statistics were performed on binned data. Additionally, for data represented by violin plots, outliers were removed using the ROUT method of identifying outliers. However, all statistics were performed on the raw data before outlier removal. Significance of all results is reported as follows: ns, not significant; $* p<0.05$; $* * p<$ $0.01 ; * * * p<0.0001$.

\section{Code accessibility}

All MATLAB code used for $\mathrm{Ca}^{2+}$ and super-resolution imaging analysis is available on request. All code was run on Windows 7 and Windows 10 operating systems. 


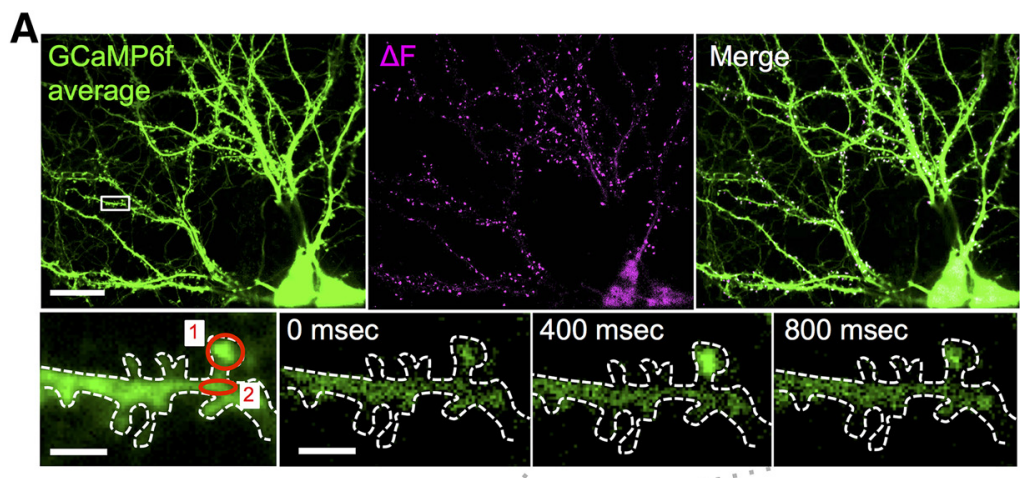

B

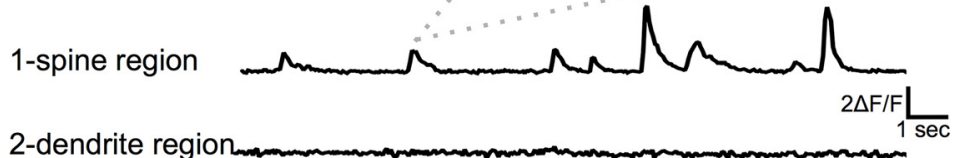

C
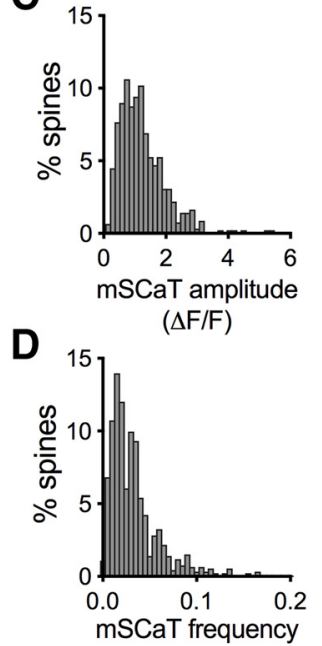

$(\mathrm{Hz})$
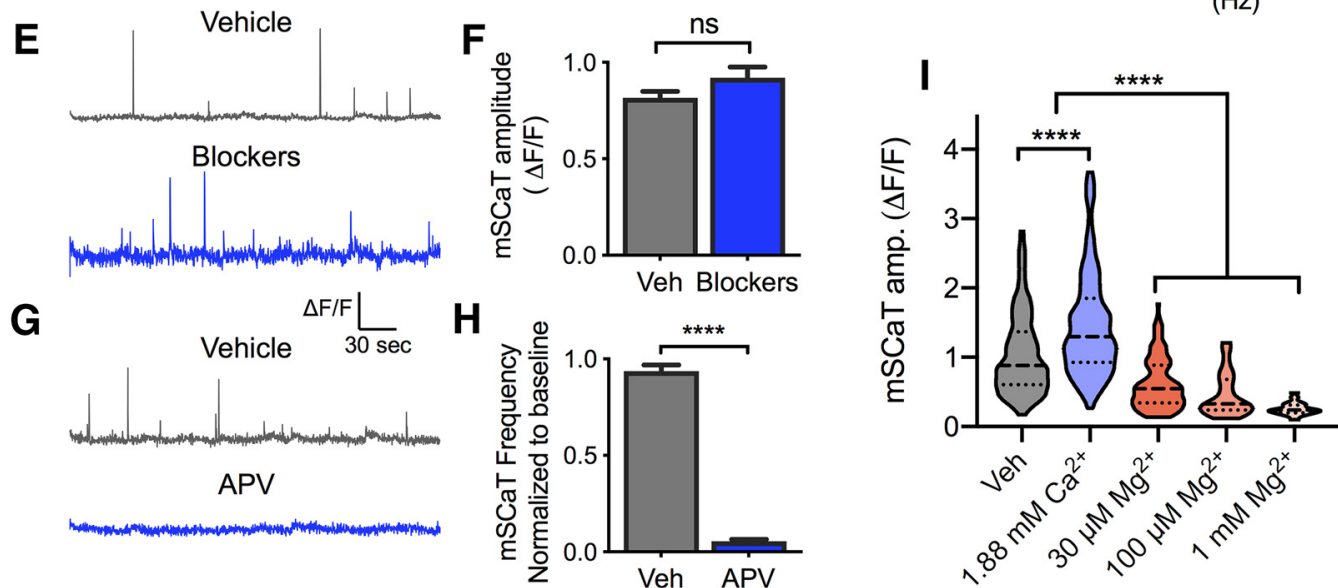

Figure 1. mSCaTs measured by GCaMP6f imaging reflect NMDAR activation at individual synapses following spontaneous single vesicle release. $\boldsymbol{A}$, Cultured hippocampal neuron infected with AAV-GCaMP6f. Left, GCaMP6f average (green); middle, $\Delta \mathrm{F}$ calculated by subtracting GCaMP6f average from GCaMP6f maximum projection (magenta); right, merge of GCaMP6f average (green) and $\Delta \mathrm{F}$ (magenta). Bottom, Zoom in of boxed spine from cell in top left. First panel is GCaMP6f average, the 2nd through 4th panels are individual frames showing a single mSCaT at 0,400 , and $800 \mathrm{~ms}$, respectively. Red circles indicate ROls for data traces shown in $\boldsymbol{B}$. $\boldsymbol{B}, \Delta \mathrm{F} / \mathrm{F}$ traces from spine and dendrite regions circled in red in $\boldsymbol{A}$. $\boldsymbol{C}$, Frequency histogram of mSCaT amplitude for individual synapses across 923 spines from 8 cells. $\boldsymbol{D}$, Frequency histogram of mSCaT frequency for individual synapses across 923 spines from 8 cells. $\boldsymbol{E}$, Representative GCaMP6f traces demonstrating that treatment with ryanodine, thapsigargin, DNQX, and nifedipine (blockers) did not alter mSCaT amplitude compared with vehicle treatment. $\boldsymbol{F}$, Quantification of effect of blockers on mSCaT amplitude compared with vehicle treatment revealed that blockade of non-NMDAR sources of $\mathrm{Ca}^{2+}$ did not impact mSCaT amplitude. G, Representative GCaMP6f traces demonstrating that treatment with APV eliminated mSCaTs compared with vehicle treatment. $\boldsymbol{H}$, Treatment with APV eliminates $94 \%$ of events. $\boldsymbol{I}$, Raising extracellular $\mathrm{Ca}^{2+} \mathrm{causes}^{2}$ increased mSCaT amplitude, whereas application of $30 \mu \mathrm{M}, 100 \mu \mathrm{M}$, and $1 \mathrm{~mm} \mathrm{Mg}^{2+}$ reduce mSCaT amplitude. For example traces, see Extended Data Figure 1-1. ns, not significant; $* * * * p<0.0001$.

\section{Results}

\section{Measuring NMDAR-mediated $\mathrm{Ca}^{2 \pm}$ transients with GCaMP6f at individual synapses}

To assess NMDAR activation by spontaneous neurotransmitter release, we sparsely infected dissociated rat hippocampal neurons at the time of plating with AAV expressing GCaMP6f, and imaged neurons at 19-22 DIV (unless otherwise noted) in ACSF containing $0 \mathrm{~mm} \mathrm{Mg}^{2+}$, and $1 \mu \mathrm{M}$ TTX to block APs. Clear mSCaTs were detected in individual spines (Fig. 1A) that were well isolated within spines and did not correspond to increases in $\mathrm{Ca}^{2+}$ in dendrites (Fig. 1B). Though $\mathrm{Ca}^{2+}$ influx through NMDARs can lead to $\mathrm{Ca}^{2+}$ release from intracellular stores (Reese and Kavalali, 2015), we found that mSCaT amplitude in spines were unaffected by ryanodine and thapsigargin to prevent $\mathrm{Ca}^{2+}$-induced $\mathrm{Ca}^{2+}$ release (CICR) from internal stores (vehicle: $0.8164 \pm 0.032 \Delta \mathrm{F} / \mathrm{F}, n=277$ spines $/ 7$ neurons; blockers: $0.9206 \pm 0.055 \Delta \mathrm{F} / \mathrm{F}$, mean $\pm \mathrm{SEM}, n$ $=239 / 6 ; p=0.095$, unpaired $t$ test; Fig. 1E,F). Despite this, to ensure isolation of the NMDAR component of spine $\mathrm{Ca}^{2+}$ changes, all experiments were done in the presence of DNQX to block AMPARs, nifedipine to block L-type $\mathrm{Ca}^{2+}$ channels, and previously mentioned CICR blockers. Application of the NMDAR antagonist APV nearly eliminated all events (post-treatment event frequency normalized to baseline: vehicle: $0.9369 \pm 0.03228, n=495 / 10$, 
APV: $0.05541 \pm 0.008763, n=197 / 5 ; p<0.0001$, unpaired $t$ test; Fig. $1 G, H)$, confirming that the observed $\mathrm{Ca}^{2+}$ transients were NMDAR-mediated.

To confirm that our measurement is sensitive to changes in NMDAR activation, we tested the dynamic range of GCaMP6f imaging at individual synapses. We reasoned that if the indicator is not near saturation, then increases in extracellular $\mathrm{Ca}^{2+}$ will produce approximately proportional increases in fluorescence intensity $(F)$, otherwise very large $\mathrm{Ca}^{2+}$ influx will saturate the indicator and result in a disproportionately low $\Delta \mathrm{F} / \mathrm{F}$. To test this, we raised the concentration of $\mathrm{Ca}^{2+}$ in the ACSF from 1.5 to $1.88 \mathrm{~mm}$ and measured $\mathrm{mSCaT}$ amplitude. We observed a significant increase in the average $\mathrm{mSCaT}$ amplitude (vehicle: $1.138 \pm 0.031 \Delta \mathrm{F} / \mathrm{F}, n=611 / 17 ; 1.88 \mathrm{mM} \mathrm{Ca}^{2+}$ : $1.56 \pm 0.051 \Delta \mathrm{F} / \mathrm{F}, n=377 / 4 ; p<0.0001$, KruskalWallis; Fig. 1/, and Extended Data Fig. 1-1), confirming that under our experimental conditions there was sufficient upper range to detect larger mSCaTs. To probe for sensitivity to smaller responses, and establish a lower limit to our detection, we added extracellular $\mathrm{Mg}^{2+}$ to block NMDAR channels, which we predicted would decrease the amplitude of mSCaTs. The $\mathrm{IC}_{50}$ of $\mathrm{Mg}^{2+}$ at a typical resting potential of $-60 \mathrm{mV}$ is $\sim 20 \mu \mathrm{M}$ for NMDARs (Kuner and Schoepfer, 1996). Increasing the extracellular $\mathrm{Mg}^{2+}$ concentration from 0 to $30 \mu \mathrm{M}$ caused a significant decrease of close to $50 \%$ in the range of mSCaT amplitudes, as expected (vehicle: $1.138 \pm 0.031$ $\Delta \mathrm{F} / \mathrm{F}, n=611 / 17 ; 30 \mu \mathrm{M}: 0.697 \pm 0.046 \Delta \mathrm{F} / \mathrm{F}, n=122 / 7$; $p<0.0001$, Kruskal-Wallis; Fig. 1/ and Extended Data Fig. 1-1), confirming that there is sufficient sensitivity to detect smaller mSCaTs. Additionally, the sensitivity of the events to $\left[\mathrm{Mg}^{2+}\right]$ further confirms that mSCaTs reflect the amount of NMDAR activation. To evaluate the lower limit of $\mathrm{mSCaT}$ detection, we raised the $\mathrm{Mg}^{2+}$ concentration again, to $100 \mu \mathrm{M}$ and $1 \mathrm{~mm}$. Both concentrations decreased mSCaT amplitude (100 $\mu \mathrm{m}: 0.513 \pm 0.049 \Delta \mathrm{F} / \mathrm{F}$, $n=66 / 5 ; 1 \mathrm{~mm}: 0.31 \pm 0.057 \Delta \mathrm{F} / \mathrm{F}, n=20 / 4 ; p<0.0001$, Kruskal-Wallis; Fig. 1/ and Extended Data Fig. 1-1) and decreased mSCaT frequency (hertz; vehicle: $0.04 \pm$ $0.002, n=414 / 3 ; 100 \mu \mathrm{M}: 0.005 \pm 0.0001, n=132 / 5 ; 1$ mм: $0.0004 \pm 0.0002, n=89 / 4 ; p<0.0001$, KruskalWallis). Interestingly, although the above $\mathrm{Mg}^{2+}$ experiments were performed in the absence of DNQX, we observed the same effects of $\mathrm{Mg}^{2+}$ in the presence of DNQX (data not shown).

Together, these observations suggest that under more physiologic conditions, AMPAR activation after spontaneous release does not alone cause sufficient depolarization to relieve the $\mathrm{Mg}^{2+}$ block on the NMDARs. This decrease in mSCaT frequency suggests that either a subset of events are too small to detect when $\mathrm{Mg}^{2+}$ is present, or that some synaptic NMDARs are effectively entirely blocked by $\mathrm{Mg}^{2+}$ at these concentrations. Measuring the distribution of individual mSCaTs in elevated $\mathrm{Mg}^{2+}$ revealed a lower $\Delta F / F$ limit of $0.1 \Delta F / F$, the amplitude of the smallest events observed, below which mSCaTs cannot be reliably detected. While we cannot deduce the true amount of $\mathrm{Ca}^{2+}$ influx from the GCaMP $6 f \Delta F / F$ because of the nonlinearities inherent in the imaging approach, these data together indicate a large dynamic range of the reporter, and suggest it provides an acceptable readout of the strength of NMDAR activation at individual synapses. To ensure that NMDARs were minimally blocked, all other experiments were performed in the absence of added $\mathrm{Mg}^{2+}$.

Critically, we observed that mSCaT amplitudes and frequencies were highly variable between synapses (Fig. $1 C, D$ ) with population inter-spine Cs of 0.64 and 0.99 , respectively. Interestingly, the mean within-spine coefficient of variation (CV) for event amplitude was also quite high, nearly at the level as what was observed between spines $(0.57 \pm 0.01 n=700 / 10$, at least 3 events per spine). This variability was not because of differences in co-agonist availability, as treatment with $100 \mu \mathrm{M}$ D-serine actually increased intra-spine mSCaT variability (mean CV normalized to baseline: vehicle: $1.05 \pm 0.05, n=159 / 4$; D-serine: $1.28 \pm 0.05, n=252 / 4 ; p=0.002$, unpaired $t$ test). We therefore asked what synaptic or receptor properties could underlie this high degree of variability in receptor activation by spontaneous release both within and between synapses.

\section{GluN2B-NMDARs mediate the majority of the NMDAR-dependent $\mathrm{Ca}^{2 \pm}$ influx by spontaneous release}

A critical regulator of NMDAR function and downstream signaling is the composition of its GluN2 subunit. Specifically, GluN2B-NMDARs are important mediators of synaptic plasticity (Foster et al., 2010; Sanhueza et al., 2011; Shipton and Paulsen, 2014), which makes them well suited to mediate the plasticity induced by changes in spontaneous NMDAR activation. Therefore, we asked what proportion of mSCaTs in mature synapses is comprised of GluN2B-NMDARs activation. To address this, we imaged GCAMP6f before and after bath application of the GluN2B-specific antagonist ifenprodil (6 $\mu \mathrm{M})$, or vehicle (Fig. $2 A, B$ ). Ifenprodil at this concentration is expected to nearly completely block GluN2B-NMDARs, block $\sim 20 \%$ of GluN2A/B-NMDARs, and have almost no effect on GluN2A-NMDARs (Stroebel et al., 2014). In our cells, treatment with ifenprodil caused a $\sim 35 \%$ reduction in mSCaT amplitude (normalized amplitude: vehicle: 0.977 $\pm 0.015, n=1204 / 20$; ifenprodil: $0.622 \pm 0.017, n=$ 572/10; $p<0.0001$, Kruskal-Wallis; Fig. 2C,E). Interestingly, we observed a dramatic reduction in mSCaT frequency following application of ifenprodil, with $\sim 30 \%$ of synapses completely silenced by ifenprodil application and an $\sim 81 \%$ reduction in overall mSCaT frequency [normalized frequency: vehicle: $0.82 \pm 0.022, n=961 / 20$; ifenprodil (3 weeks): $0.138 \pm 0.006, n=788 / 10 ; p<$ 0.0001 , Kruskal-Wallis; Fig. 2D,F]. The dramatic drop in event frequency along with complete blockade of events at half of the synapses suggests a significant portion of mSCaTs are entirely GluN2B-NMDAR mediated, and that GluN2A-NMDARs contribute very little to NMDARmediated $\mathrm{Ca}^{2+}$ influx in response to spontaneous release.

Additionally, because GluN2B-NMDAR levels are developmentally regulated in some brain areas (Chen et al., 2000; Barth and Malenka, 2001; Ritter et al., 2002; 
A

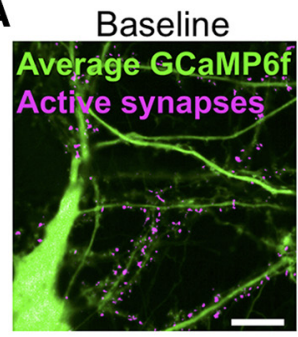

Baseline

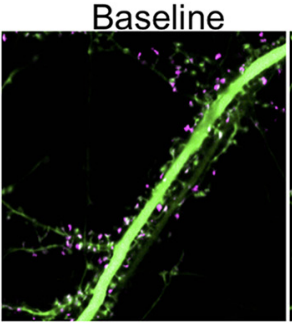

B

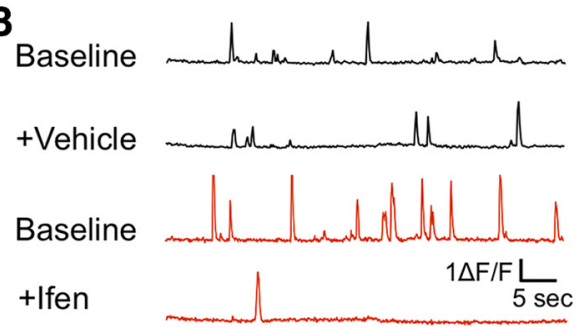

C
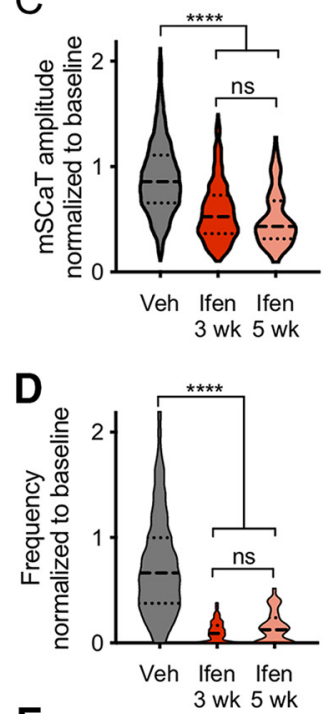

E

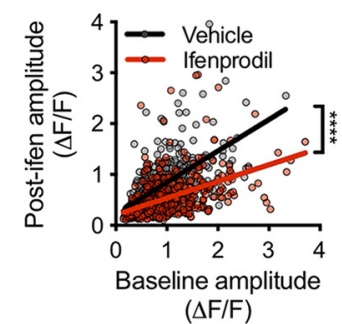

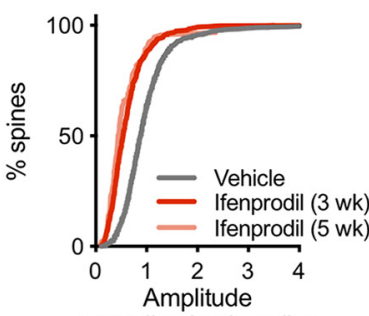

normalized to baseline

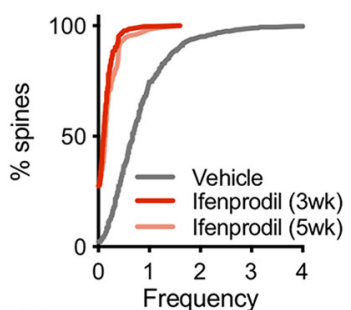

F normalized to baseline

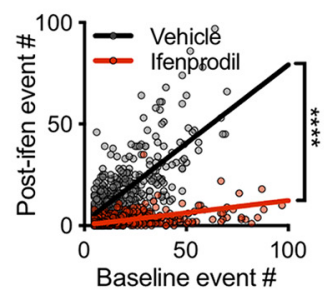

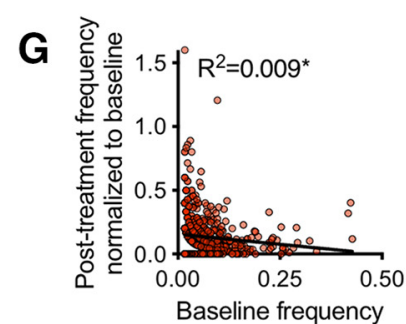

$(\mathrm{Hz})$

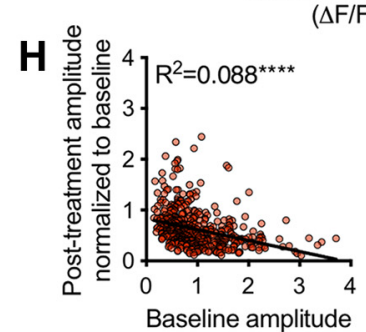

$(\Delta F / F)$

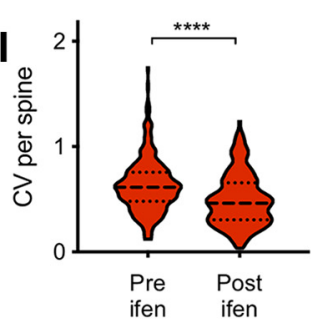

ifen ifen

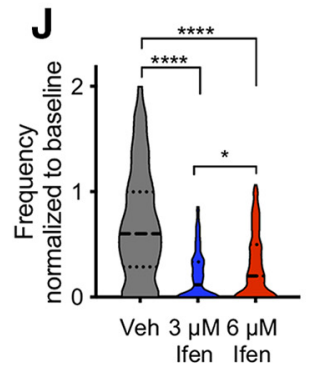

$\mathbf{L}$

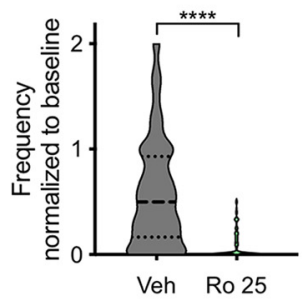

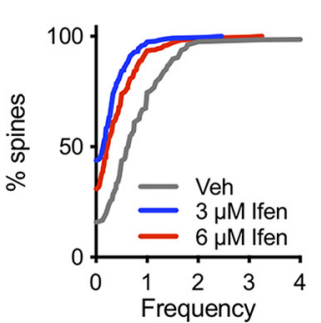

normalized to baseline

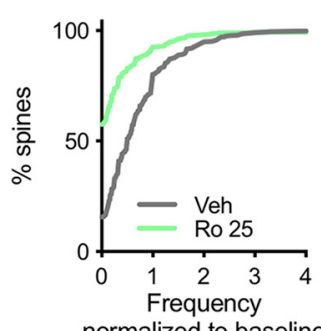

K

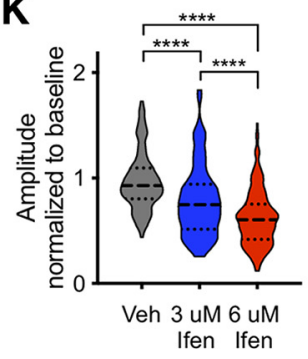

M

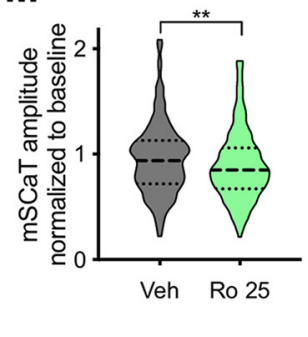

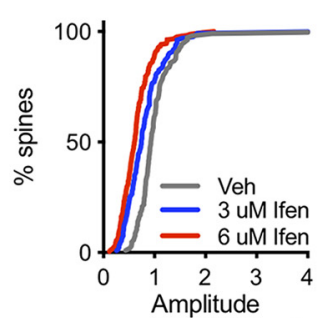

normalized to baseline

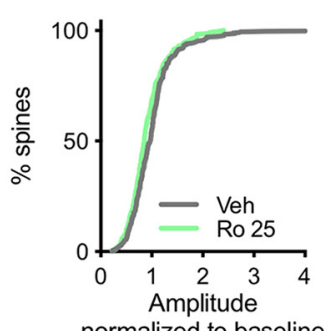

Figure 2. GluN2B-NMDARs mediate majority of response to spontaneous glutamate release. A, Average projection of GCaMP6f 
continued

(green) at baseline and following treatment with vehicle or ifenprodil. Active synapses are shown in magenta. Following treatment with ifenprodil there is a clear reduction in the number of active spines compared with baseline. Scale bar, $10 \mu \mathrm{m}$. $\boldsymbol{B}$, Example traces from spines treated with either vehicle (black) or ifenprodil (red). $\boldsymbol{C}$, Ifenprodil treatment leads to a reduction in mSCaT amplitude at both 3 and 5 weeks. Outliers removed for data display. Solid line represents median and dashed lines indicate 1st and 3rd quartile. Second plot is cumulative probability for all spines. For immunoblotting data, see Extended Data Figure 2-1 and Extended Data Figure 2-2. $\boldsymbol{D}$, Ifenprodil treatment leads to a reduction in mSCaT frequency at both 3 and 5 weeks. Outliers removed for data display. Solid line represents median and dashed lines indicate 1st and 3rd quartile. Second plot is cumulative probability for all spines. $\boldsymbol{E}$, Posttreatment $\mathrm{mSCaT}$ amplitude versus baseline mSCaT amplitude has a slope of $0.6167 \pm 0.033$ for vehicle-treated cells and $0.327 \pm$ 0.025 for ifenprodil-treated cells and these slopes are significantly different $(p<0.0001)$. Post-treatment mSCaT amplitude is correlated with baseline amplitude for both vehicle-treated synapses $\left(R^{2}=0.42, p<0.0001\right)$ and ifenprodil-treated synapses $\left(R^{2}=0.22, p<0.0001\right)$. $\boldsymbol{F}$, Baseline mSCaT frequency versus post-treatment mSCaT frequency reveals that nearly all synapses show a reduction in event number with ifenprodil treatment (vehicle: slope $=0.7984 \pm 0.02368$; ifenprodil: slope $=0.108 \pm 0.005452 ; p<$ 0.0001). Post-treatment mSCaT amplitude is correlated with baseline amplitude for both vehicle-treated synapses $\left(R^{2}=0.56, p<\right.$ $0.0001)$ and ifenprodil-treated synapses $\left(R^{2}=0.31, p<0.0001\right)$. $\mathbf{G}$, Normalized amplitude post-ifenprodil treatment is negatively correlated with baseline amplitude. $\boldsymbol{H}$, Normalized mSCaT frequency post-ifenprodil treatment is negatively correlated with baseline mSCaT frequency. I, Within spine CV decreases following ifenprodil treatment. $\boldsymbol{J}$, Three micromolar ifenprodil (blue) treatment leads to a significant reduction in $\mathrm{mSCaT}$ frequency. Outliers removed for data display in violin plots. Second plot is cumulative probability for all spines. $\boldsymbol{K}$, Three micromolar ifenprodil (blue) treatment leads to a significant reduction in mSCaT amplitude. Outliers removed for data display in violin plots. Second plot is cumulative probability for all spines. $\boldsymbol{L}$, Ro 25-6981 (green) treatment leads to a significant reduction in mSCaT frequency. Outliers removed for data display in violin plots. Second plot is cumulative probability for all spines. $\boldsymbol{M}$, Ro 25-6981 (green) treatment leads to a significant reduction in mSCaT amplitude. Outliers removed for data display in violin plots. Second plot is cumulative probability for all spines. ns, not significant; $* p<0.05 ; * * p<0.01 ; * * * * p<0.0001$.

Yashiro and Philpot, 2008), we imaged neurons at 5 weeks, to assess whether the large effect of ifenprodil on mSCaT frequency was because of the age of the cells. We found a significant reduction in amplitude and frequency with ifenprodil in 5 week cells [normalized amplitude (5 weeks): $0.608 \pm 0.049, n=94 / 9$; normalized frequency (5 weeks): $0.196 \pm 0.022, n=130 / 9 ; p<0.0001$, KruskalWallis], and no difference between the effects of ifenprodil on 3 or 5 -week-old neurons (amplitude: $p=0.546$, Kruskal-Wallis; frequency: $p=0.0247$, Kruskal-Wallis; Fig. $2 C, D$ ), indicating that GluN2B-NMDARs contribute significantly to spontaneous events in mature cultured hippocampal neurons of different ages. It is conceivable that expression of GCaMP6f, by chronic alteration of $\mathrm{Ca}^{2+}$ buffering in the cell, could lead to abnormal expression of GluN2 subunits and prevent the typical developmental shift in the ratio of GluN2B:GluN2A subunits (Yashiro and Philpot, 2008). To test this, we examined subunit expression levels at 12 and 19 DIV in control cultures, or in cultures that had been infected with GCaMP6f or GFP. In uninfected cultures, we observed the expected decrease in the ratio, and this decrease was unaltered in either virus-infected condition (Extended Data Fig. 2-1C,D and Extended data Fig. 2-2). We then examined ifenprodil sensitivity in neurons that were only transiently transfected with GCaMP6f, rather than infected. We found that neurons that expressed GCaMP6f from 18-21 DIV showed the same high ifenprodil sensitivity as those chronically expressing via virus infection (Normalized amplitude: Veh-infected: $0.98 \pm 0.016, n=$ 1204/20; Ifen-infected: $0.622 \pm 0.017, n=572 / 10$; Vehtransfected: $1.07 \pm 0.035, n=103 / 4$; Ifen-transfected: $0.633 \pm 0.051, n=81 / 4 ; p<0.0001$, one-way ANOVA; Normalized frequency: Veh-infected: $0.82 \pm 0.022, n=$ 961/20; Ifen-infected: $0.137 \pm 0.006, n=788 / 10$; Vehtransfected: $0.855 \pm 0.063, n=110 / 4$; Ifen-transfected: $0.20 \pm 0.048, n=142 / 4 ; p<0.0001$, one-way ANOVA;
Extended Data Fig. 2-1A,B). These observations suggest that chronic GCaMP6f expression does not alter subunit expression.

Returning to further analysis of virally infected neurons, we also observed that synapses with larger mSCaTs at baseline were associated with a greater effect of ifenprodil on mSCaT amplitude $\left(R^{2}=0.089, p<0.0001, n=\right.$ 544/10; Fig. 2G), and spines with higher mSCaT frequency at baseline tended to have a larger portion of events blocked with ifenprodil $\left(R^{2}=0.012, p=0.003, n=\right.$ 751/10; Fig. $2 H$ ). Thus, more active synapses, with more NMDAR activation, have a larger contribution of GluN2Bcontaining NMDARs to their events. Because GluN2BNMDARs have longer open times and lower open probability than GluN2A-NMDARs, it is likely that there is increased variability in $\mathrm{Ca}^{2+}$ influx through GluN2BNMDARs (Santucci and Raghavachari, 2008). Indeed, we observed that mSCaT amplitude variance was slightly reduced following ifenprodil treatment (baseline CV: 0.633 \pm 0.02; post-ifenprodil CV: $0.495 \pm 0.016 ; n=225, p<$ 0.0001 , paired $t$ test; Fig. 2l), however, this could at least be partially because of the large reduction in the number of events. Overall, NMDAR subtype may contribute to the high degree of variability in NMDAR-mediated $\mathrm{Ca}^{2+}$ influx. Together, these results not only indicate that GluN2BNMDARs contribute to events at these synapses, but also that a significant portion of mSCaTs are largely GluN2BNMDAR mediated.

Finally, because $6 \mu \mathrm{M}$ ifenprodil can to some extent block NMDARs subtypes other than GluN1/GluN2B diheteromeric NMDARs (Hatton and Paoletti, 2005; Hansen et al., 2014; Stroebel et al., 2018), we also treated cells with $3 \mu \mathrm{M}$ ifenprodil or $1 \mu \mathrm{M}$ Ro 25-6981. At $3 \mu \mathrm{M}$, ifenprodil still is expected to block nearly all GluN2BNMDARs, but the off-target effects on GluN2A-NMDARs and GluN2A/B-NMDARs should be reduced (Hatton and Paoletti, 2005; Hansen et al., 2014; Stroebel et al., 2018). 
In a separate set of experiments in which matched neurons were treated with vehicle, $3 \mu \mathrm{M}$, or $6 \mu \mathrm{M}$ ifenprodil, each concentration strongly reduced $\mathrm{mSCaT}$ frequency (Fig 2J). In fact, the effect at $3 \mu \mathrm{M}$ was slightly stronger (vehicle: $0.829 \pm 0.0974, n=126 / 8$; ifen $3 \mu \mathrm{M}$ : $0.251 \pm$ $0.0229, n=235 / 10$; ifen $6 \mu \mathrm{M}: 0.379 \pm 0.0257, n=349 / 9$; $p<0.0001$, Kruskal-Wallis; Fig. 2J). This is consistent with the idea that the effect of ifenprodil on $\mathrm{mSCaT}$ frequency is because of blockade of GluN2B-NMDARs rather than other NMDAR subtypes. Interestingly, although there was a significant decrease in $\mathrm{mSCaT}$ amplitude with $3 \mu \mathrm{m}$ ifenprodil, it was less pronounced than the effect of $6 \mu \mathrm{M}$ ifenprodil on amplitude (vehicle: $1.022 \pm$ $0.0484, n=106 / 8$; ifen $3 \mu \mathrm{M}$ : $0.8119 \pm 0.0385, n=$ 132/10; ifen $6 \mu \mathrm{M}$ : $0.647 \pm 0.0203, n=241 / 9 ; p<0.0001$, Kruskal-Wallis; Fig. $2 K$ ). The difference in amplitude reduction with 3 and $6 \mu \mathrm{m}$ ifenprodil suggests that some of the reduction in mSCaT amplitude observed with $6 \mu \mathrm{M}$ ifenprodil may be because of blockade of GluN2A/B or GluN2A-NMDARs at individual synapses.

We next explored the effects of Ro 25-6981, a related compound which is more specific than ifenprodil for GluN2B-NMDARs compared with GluN2A-NMDARs (Fischer et al., 1997), though unfortunately the effects of Ro 25-6981 on triheteromeric receptors are unknown. Treatment with Ro 25-6981 had a similar effect on mSCaT frequency as ifenprodil (vehicle: $0.69 \pm 0.035, n=435$ / 17; Ro 25-6981: $0.283 \pm 0.027, n=547 / 18 ; p<0.0001$, unpaired $t$ test; Fig. $2 L$ ), thus further supporting the role of GluN2B-NMDARs in responding to spontaneous glutamate release. Similar to the effect of $3 \mu \mathrm{M}$ ifenprodil, the reduction in $\mathrm{mSCaT}$ amplitude was smaller than observed with $6 \mu \mathrm{M}$ ifenprodil while still significant (vehicle: $1.013 \pm$ 0.025, $n=367 / 17$; Ro 25-6981: $0.917 \pm 0.025, n=$ 232/18; $p=0.009$, unpaired $t$ test; Fig. 2M). Together, the reduction in mSCaT frequency by $3 \mu \mathrm{M}$ ifenprodil or Ro 25-6081 confirms that GluN2B-containing NMDARs are the primary NMDAR subtype activated by spontaneous release at nearly all synapses in this preparation.

\section{NMDAR activation is independent of spine and synapse size}

Whereas GluN2B-NMDARs contribute to variability of mSCaT amplitude within synapses, other parameters may contribute to variability between synapses. Although there is evidence that the number of NMDARs present in the PSD (Kharazia and Weinberg, 1999; Takumi et al., 1999; Shinohara et al., 2008; Chen et al., 2015) as well as the magnitude of their activation by evoked release is not related to spine size (Nimchinsky et al., 2004), there is a strong relationship between spine size and the amount of activation of other receptor types, particularly AMPARs (El-Husseini et al., 2000; Matsuzaki et al., 2004; Araki et al., 2015). Because NMDAR activation by spontaneous release may be regulated distinctly from NMDAR activation by evoked release, we tested the relationship between $\mathrm{mSCaTs}$ and spine size. We measured spine area at individual synapses based on post- $\mathrm{Ca}^{2+}$ imaging $z$-stacks (Fig. $3 A, B$ ). Spine area ranged from 0.163 to $1.411 \mu \mathrm{m}^{2}$ with a mean spine area of $0.639 \pm 0.009 \mu \mathrm{m}^{2}$.
By matching spine area to $\mathrm{mSCaT}$ data for each synapse, we found that mean mSCaT amplitude per spine was weakly negatively correlated with spine area for spontaneous release events $\left(R^{2}=0.008, p=0.028, n=628 / 10\right)$. A similar result was seen with binned spine area data to reduce overpowering the analysis $\left(R^{2}=0.256, p=0.023\right.$, $n=20 / 10$; Fig. $3 C$ ). However, this negative correlation is likely because of smaller proportional $\mathrm{Ca}^{2+}$ influx in larger spines rather than differences in the amount of NMDAR activation (Nimchinsky et al., 2004). Overall, this suggests that NMDAR activation does not substantially scale with spine size.

GluN2B-NMDARs have been shown to exit synapses that have undergone LTP (Dupuis et al., 2014) and those synapses tend to be larger (Matsuzaki et al., 2001, 2004; Malenka and Bear, 2004). Therefore, it is possible there is a smaller contribution of GluN2B-NMDARs at larger spines. To address this, we examined the correlation between the effect of ifenprodil on mSCaTs at individual synapses and spine area. We found that spine area did not correlate with the magnitude of ifenprodil blockade on amplitude or frequency of mSCaTs (normalized amplitude: raw: $R^{2}=0.006, p=0.388, n=127 / 6$; binned: $R^{2}$ $=0.050, p=0.342, n=20 / 6$; normalized frequency: $R^{2}=$ 0.012, $p=0.109, n=221 / 6$; binned: $R^{2}=0.059, p=$ $0.298, n=20 / 6$; Fig. 3D). These data suggest that the contribution of GluN2B-NMDARs per synapse is not related to spine area.

Although spine size is often related to synapse size (Harris et al., 2014), the PSD is much smaller than the spine itself, and is a highly dynamic structure that is strongly correlated to the size of the active zone across the cleft (Harris and Stevens, 1989; Schikorski and Stevens, 1997; Inoue and Okabe, 2003; MacGillavry et al., 2013). Thus, to measure the area of the PSD itself using super-resolution microscopy, we turned to dSTORM imaging of the postsynaptic protein Shank. We matched mSCaT data to super-resolved PSDs for 47 synapses from 6 neurons that were subjected to live GCaMP6f imaging followed by fixation and anti-Shank immunocytochemistry and dSTORM imaging (Fig. $3 E-H$ ). The mean PSD area was $0.060 \pm 0.006 \mu \mathrm{m}^{2}$, which is near the mean reported by electron microscopy of hippocampal synapses (mean $\sim 0.069 \mu \mathrm{m}^{2}$; Harris and Stevens, 1989; Schikorski and Stevens, 1997; Shinohara et al., 2008). We found that $\mathrm{mSCaT}$ amplitude was not correlated with PSD area $\left(R^{2}=0.013, p=0.452, n=47 / 6\right.$; Fig. $\left.3 /\right)$.

Together, this set of observations indicates that the size of the activated pool of NMDARs at individual synapses is independent of synapse size and that variability in the magnitude of NMDAR activation observed between synapses for spontaneous release events is controlled by factors other than the size of the synapse itself.

\section{mSCaT amplitude is correlated with synapse distance from the soma}

Another potential source of variability in $\mathrm{mSCaT}$ amplitude and frequency is the position of the synapse within the dendritic tree. In fact, NMDAR-mediated $\mathrm{Ca}^{2+}$ influx 
A

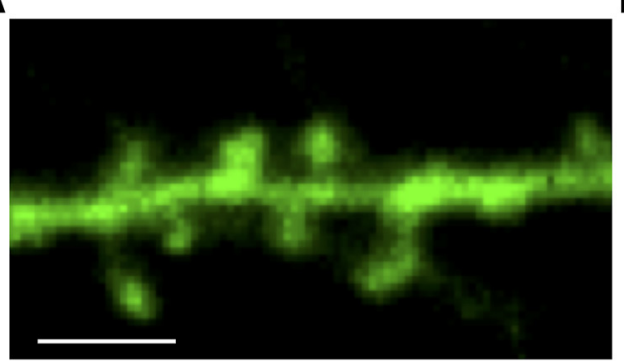

B

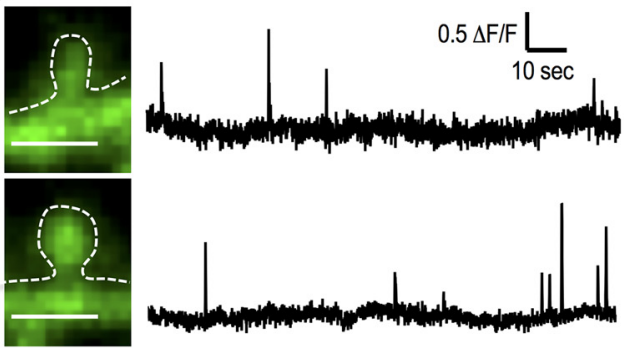

C
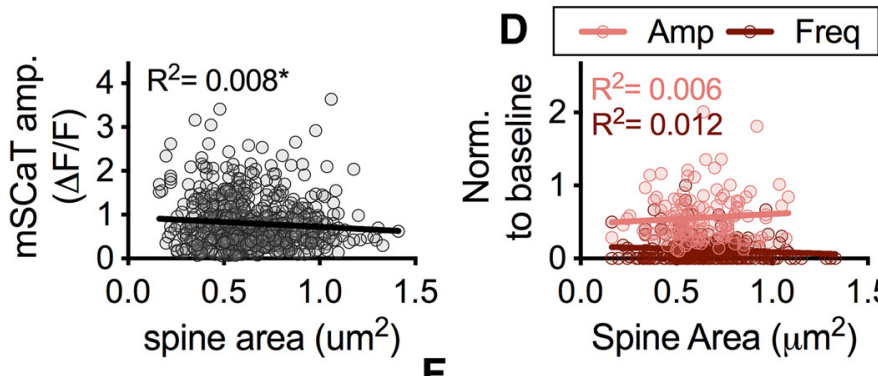

E

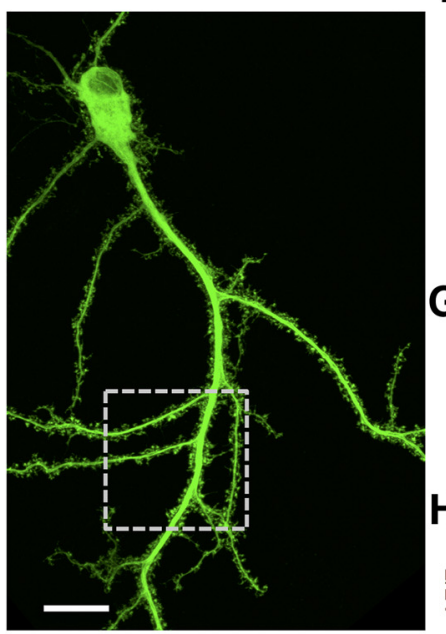

$F$
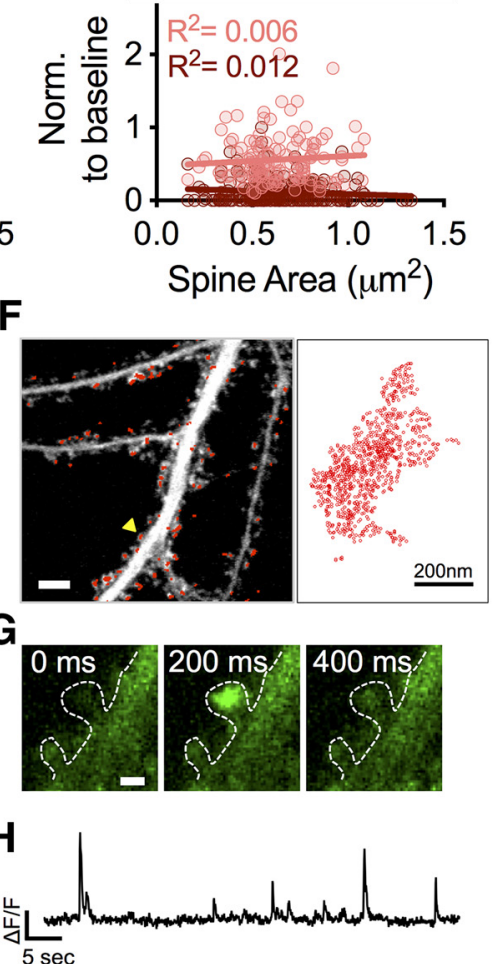

I

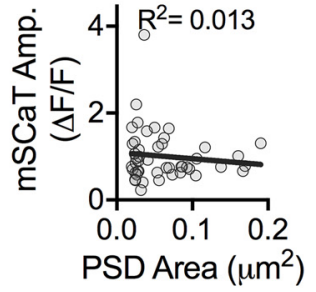

Figure 3. NMDAR activation is independent of spine area. $\boldsymbol{A}$, Example stretch of dendrite from post-Ca ${ }^{2+}$ imaging GCaMP6f $z$-stack. Scale bar, $10 \mu \mathrm{m}$. $\boldsymbol{B}$, Zoom in of spines indicated by white arrowheads from $\boldsymbol{A}$ paired with their respective $\mathrm{Ca}^{2+}$ traces. Scale bar, $1 \mu \mathrm{m}$. C, mSCaT amplitude weakly, negatively correlates with spine area. First plot is all spines, second plot binned data. $\boldsymbol{D}$, Effect of ifenprodil on amplitude (light red) and frequency (dark red) does not correlate with spine area. Left plot is all spines; right plot is binned data. $\boldsymbol{E}$, GCaMP6f max projection acquired directly following $\mathrm{Ca}^{2+}$ imaging (scale bar, $20 \mu \mathrm{m}$ ) of dSTORM imaged neuron. White box indicates area where super-resolution imaging was performed. $\boldsymbol{F}$, Zoom in on the region from $\boldsymbol{E}$. Max projection of GCaMP6f stack acquired at time of STORM imaging (white). Scale bar, $5 \mu \mathrm{m}$. Super-resolved shank localizations are shown in red. Right, Zoom-in of the shank localizations from the spine indicated with the yellow arrowhead. $\mathbf{G}$, Zoom-in of a mSCaT in the spine indicated by yellow arrowhead in $\boldsymbol{F} . \boldsymbol{H}, \mathrm{Ca}^{2+}$ trace from spine indicated by yellow arrowhead in $\boldsymbol{F}$. $\boldsymbol{I}$, Amplitude does not correlate with PSD area.

has been shown to be increased in synapses further from the cell body (Walker et al., 2017). We asked whether NMDAR activation by spontaneous glutamate release varies throughout the dendritic arbor as a function of distance from the soma or the number of branch points away from the soma (branch depth). We mapped neuronal morphology and identified spines along dendrites from $z$-stacks of GCaMP6f-expressing neurons using semiautomatic neuron tracing and spine detection in Imaris (Fig. $4 A-C)$ and identified spines in the traced image that had been $\mathrm{Ca}^{2+}$ imaged (Extended Data Fig. 4-1). Mean mSCaT amplitude per spine was significantly correlated with distance from the soma $\left(R^{2}=0.014, p=0.036, n=\right.$ 316/9; Fig. 4C), as expected (Walker et al., 2017). Interestingly, although branch depth was correlated with distance from the soma $\left(R^{2}=0.022, p<0.0001\right.$; data not shown), branch depth did not correlate with mean mSCaT amplitude (raw data: $R^{2}=0.0007, p=0.649, n=287 / 9$; Fig. $4 E$ ). To compare spines that are farther than $\sim 200$ $\mu \mathrm{m}$ away from the soma, we repeated these experiments using a lower-power objective to increase the field-ofview. This allowed for a larger field-of-view and measurement of distances up to $\sim 450 \mu \mathrm{m}$ away from the soma. We observed that even at these longer distances, there 


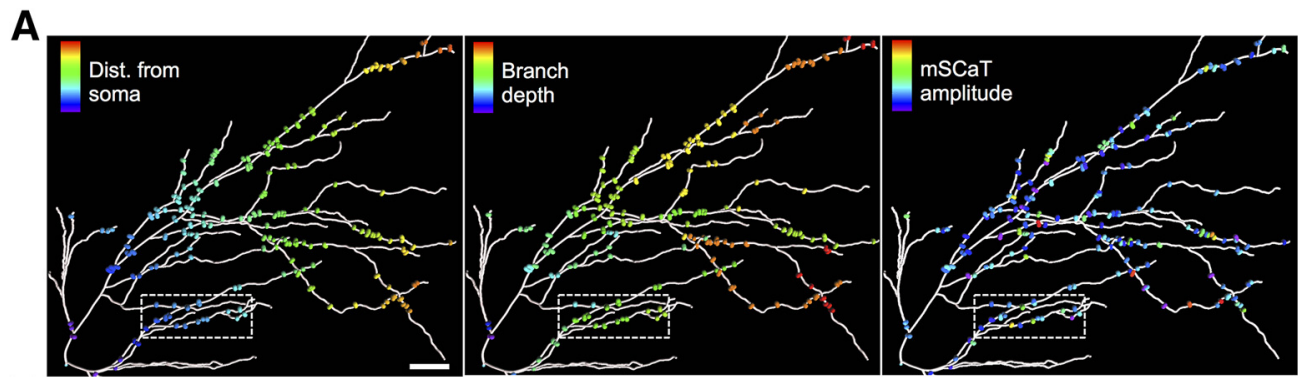

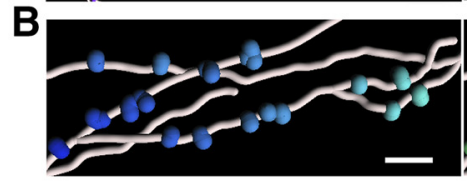

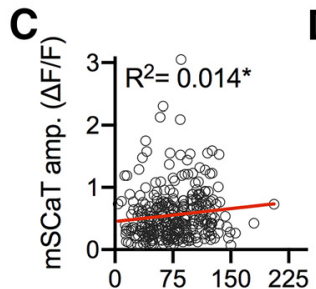

Dist. from soma $(\mu \mathrm{m})$

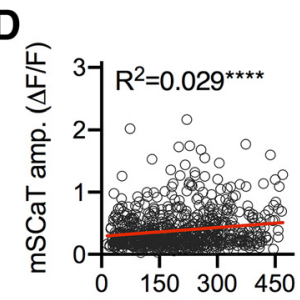

Dist. from soma $(\mu \mathrm{m})$

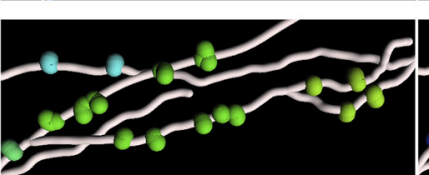

E

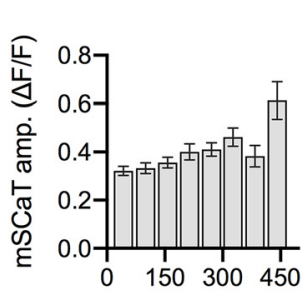

Dist. from soma $(\mu \mathrm{m})$

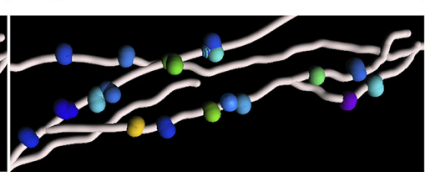

G

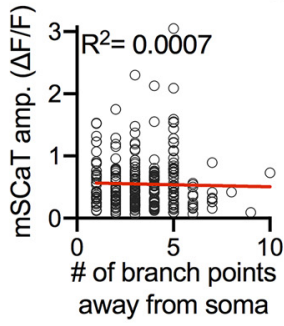

H

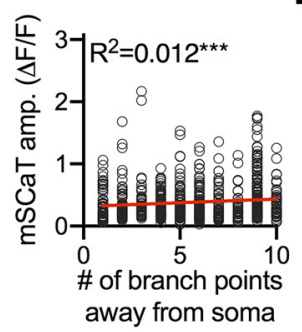

1

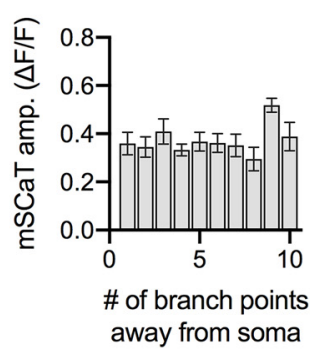

$\mathbf{F}$

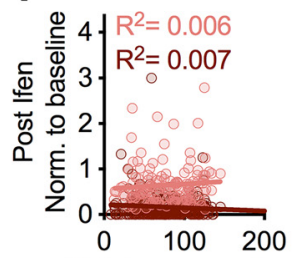

Dist. from soma $(\mu \mathrm{m})$

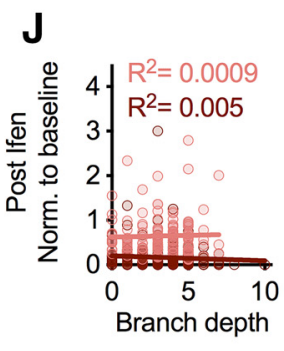

Figure 4. Mapping synapse position and mSCaT characteristics with Imaris. $\boldsymbol{A}, \mathrm{Ca}^{2+}$ imaged spines along traced dendrite from Imaris. Scale bar, $30 \mu \mathrm{m}$. First panel shows distance from soma, second panel shows branch depth, and third panel shows mSCaT amplitude. Colors are warmer as spines that are farther from the cell body, have higher branch depth, or have larger mSCaT mean amplitudes respectively. For example of Imaris tracing see Extended Data Figure 4-1. B, Zoom-in of boxed areas from $\boldsymbol{A}$. C, mSCaT amplitude did correlate with distance from soma for proximal spines. $\boldsymbol{D}$, mSCaT amplitude did correlate with distance from soma when distal spines are included. $\boldsymbol{E}$, Binned data from $\boldsymbol{D}$ demonstrating relationship between spine distance from the soma and $\mathrm{mSCaT}$ amplitude $\boldsymbol{F}$, Magnitude of ifenprodil effect on mSCaT amplitude (light red) and frequency (dark red) does not correlate with distance from the soma. G, mSCaT amplitude did not correlate with the number of branch points away from the soma the synapse is (branch depth) for proximal spines. $\boldsymbol{H}, \mathrm{mSCaT}$ amplitude does correlate with branch depth when distal spines are included. $\boldsymbol{I}$, Mean mSCaT amplitude at each branch depth. $\boldsymbol{J}$, Magnitude of ifenprodil effect on $\mathrm{mSCaT}$ amplitude (light red) and frequency (dark red) did not correlate with branch depth.

was a correlation between mSCaT amplitude and distance from soma $\left(R^{2}=0.041, p<0.0001, n=746 / 6\right.$; Fig. $4 D, E)$. Interestingly, when mSCaT amplitude was compared with branch depth at longer distances there was a correlation between mSCaT amplitude and branch depth $\left(R^{2}=0.018, p=0.0002, n=746 / 6\right.$; Fig. $\left.4 H, I\right)$. These results demonstrate that the amount of NMDAR activation following spontaneous single vesicle release is related to the synapse distance from soma, and branch depth at more distal branches.

We then further asked whether GluN2B-NMDAR content varied based on synapse position. We found no correlation between the magnitude of the effect of ifenprodil with either distance from the soma (normalized frequency: raw data: $R^{2}=0.007, p=0.237, n=209 / 9$; binned data: $R^{2}=0.036, p=0.422, n=20 / 9$; normalized amplitude: raw data: $R^{2}=0.006, p=0.367, n=132 / 9$; binned data: $R^{2}=0.034, p=0.434, n=20 / 9$; Fig. $4 F$ ) or branch depth (normalized frequency: raw data: $R^{2}=0.005$, $p=0.315, n=209 / 9$; binned data: $R^{2}=0.007, p=0.721, n$ = 209/9; normalized amplitude: raw data: $R^{2}=0.0009, p=$ 0.729, $n=20 / 9$; binned data: $R^{2}=0.00004, p=0.978, n=$ 20/9; Fig. 4J). Therefore, the amount of GluN2B-NMDAR activation is independent of synapse position within the dendritic arbor.

Overall, synapse position did loosely correlate with mSCaT amplitude, whereas spine and synapse size did not. Furthermore, the GluN2B component of mSCaTs is also independent of synapse size and position. Therefore, it likely that there are other factors besides size or 
position that are responsible for the dramatic variability in spontaneous NMDAR activation between synapses.

\section{Discussion}

In the present study, we used an all-optical approach to characterize NMDAR activation following AP-independent (spontaneous) vesicle exocytosis at individual synapses of cultured hippocampal neurons. Whereas GCaMP6f has frequently been used to measure mSCaTs (Andreae and Burrone, 2015; Sinnen et al., 2016; Tang et al., 2016; Walker et al., 2017), we clarified that it offers a large dynamic range and permits detailed analysis of the magnitude of receptor activation. Using this approach, we found that at nearly all synapses in this preparation, GluN2B-NMDARs are the major NMDAR subtype activated during spontaneous synaptic transmission. Additionally, we observed a surprising degree of variability in mSCaT amplitude both between and within synapses. In fact, the variation at single spines was comparable to the between-spine variation, which suggests that $\mathrm{Ca}^{2+}$ influx through the receptor may be surprisingly independent of synapse-specific features such as the number of receptors present at the synapse. Indeed, we demonstrated that spine size, PSD area, and synapse position have a relatively small impact on mSCaTs. Therefore, this high degree of variability is likely to be dominated by differences in release position, variations in the amount of glutamate per vesicle, and random fluctuations in channel open time.

We found that even in the absence of an AMPAR an-

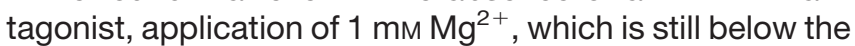
physiologic concentration, nearly eliminates all observable mSCaTs. This suggests that under more physiologic conditions, in the absence of other activity, many spontaneous release events do not lead to sufficient membrane depolarization to relieve the $\mathrm{Mg}^{2+}$ block on NMDARs, and thus result in little or no $\mathrm{Ca}^{2+}$ influx. Recent evidence demonstrates that glutamate binding to NMDARs can induce conformational changes in the receptor which lead to metabotropic signaling even in the absence of ion flux (Nabavi et al., 2013; Dore et al., 2016, 2017). Importantly, this type of NMDAR activation can mediate some forms of plasticity (Kessels et al., 2013; Aow et al., 2015; Dore et al., 2015; Stein et al., 2015; Wong and Gray, 2018). Thus, our data suggest that under physiologic conditions, effects of NMDAR activation by single-vesicle release events could be mediated principally by non-ionotropic functions rather than via $\mathrm{Ca}^{2+}$ influx.

Our data demonstrate that GluN2B-NMDARs contribute significantly to spontaneous events at all synapses and, at approximately half of synapses, are the primary NMDAR type activated by spontaneous release. This was somewhat surprising given the prevailing notion that GluN2B-NMDARs are not present at synapses after early development (Chen et al., 2000; Barth and Malenka, 2001; Ritter et al., 2002; Yashiro and Philpot, 2008). However, this is consistent with other reports (Sinnen et al., 2016; Walker et al., 2017) and a large and growing amount of evidence suggests that GluN2B-NMDARs are found at mature hippocampal synapses (Kellermayer et al., 2018) and they contribute significantly to synaptic events (Gray et al., 2011; Xiao et al., 2016; Levy et al., 2018). Thus, although it is clear that in some brain areas a developmental switch in NMDAR subtype is pronounced, in the hippocampus it is not as prominent. Spontaneous NMDAR activation has specialized functions within the synapse (Sutton et al., 2004, 2006; Kavalali et al., 2011; Andreae and Burrone, 2015), and it is possible that these functions are specifically driven by GluN2B-NMDAR activation, rather than NMDAR activation in general. It will be important to assess whether GluN2B-NMDARs are required for the downstream signaling induced by spontaneous release.

We observed not only a large contribution of GluN2BNMDARs to mSCaTs, but also a striking lack of $\mathrm{Ca}^{2+}$ influx mediated by GluN2A-NMDARs. Data regarding the contribution of NMDAR subtype to synaptic responses to spontaneous glutamate release has been mixed. In previous reports, knock-out of GluN2A-NMDARs reduced or eliminated NMDA-mEPSCs in mature midbrain synapses (Townsend et al., 2003; Zhao and Constantine-Paton, 2007), thus suggesting that GluN2A-NMDARs are the principle responders to spontaneous release. This may be a region-specific effect, or, because GluN2A-NMDARs are essential for normal development of synapses in many brain regions (Gambrill and Barria, 2011; Gray et al., 2011; Kannangara et al., 2014), it is possible that global GluN2A knock-out alters synapses in other, unexpected ways.

Although GluN2A-NMDARs did not contribute significantly to mSCaTs here, they are present at hippocampal synapses in culture and function in evoked neurotransmission (MacGillavry et al., 2013; Xiao et al., 2016; Kellermayer et al., 2018). Why are they less activated by spontaneous release? One option is that there is segregation of NMDARs such that the receptors activated by spontaneous release form a distinct pool, either a subset of or separate from those activated by evoked release. There is evidence to support this idea (Atasoy et al., 2008; Reese and Kavalali, 2016), and it possible that NMDAR subtype is specific to one pool or the other. A potential mechanism for restricting NMDAR activation is spatial segregation within the synapse. Indeed, within synapses, receptors are found to have a distinct nanoscale organization with subsynaptic high-density nanoclusters of postsynaptic proteins and AMPARs as well as GluN2BNMDARs (Fukata et al., 2013; MacGillavry et al., 2013; Nair et al., 2013). Moreover, importantly, these postsynaptic nanodomains are aligned with presynaptic evoked release sites (Tang et al., 2016). Recently it has been demonstrated that within individual synapses, GluN2Aand GluN2B-NMDARs form distinct nanodomains that differ in size, number, and internal density (Kellermayer et al., 2018), further consistent with the idea that nanoorganization could influence which receptor type is activated. Interestingly because of their biophysical properties, GluN2B-NMDARs are especially likely to be sensitive to their positioning with respect to the site of release; receptors within $\sim 50 \mathrm{~nm}$ of the site of release three times more likely to open than those located $\sim 200 \mathrm{~nm}$ from the 
site of release (Santucci and Raghavachari, 2008). Therefore, the position of release site with respect to NMDARs may restrict not just the total amount of NMDAR activation but also which synaptic NMDARs are able to be activated by spontaneous release. Further, mapping of release sites during spontaneous and evoked release revealed that these release modes display different spatial patterns within the active zone (Tang et al., 2016). Given the possibility that GluN2B-NMDARs are positioned within the synapses as to be relevant for spontaneous release, the contribution of GluN2B-NMDARs to events in mature synapses may have been underestimated because of a focus on evoked release.

In addition to GluN2B-NMDARs and GluN2A-NMDARs, mature hippocampal synapses are thought to contain a significant population of triheteromeric receptors (GluN2A) B-NMDARs; Rauner and Köhr, 2011; Paoletti et al., 2013; Tovar et al., 2013; Stroebel et al., 2018). Unfortunately, these are difficult to study in situ because of a lack of specific pharmacological agents. Based on doseinhibition curves for ifenprodil for the different receptor subtypes, the $6 \mu \mathrm{M}$ ifenprodil concentration used here is likely to have blocked nearly all GluN2B-NMDAR activation Iwith little impact on GluN2A-NMDARs, but also inhibited $\sim 20 \%$ of GluN2A/B-NMDAR-mediated responses (Paoletti, 2011; Hansen et al., 2014; Stroebel et al., 2014). This probable effect on triheteromeric receptors may indicate that these receptors contribute to some spontaneous events. Indeed, the reduction in mSCaT amplitude was less dramatic with $3 \mu \mathrm{m}$ ifenprodil and Ro 25-6981, though still present. The consistent effects with these three antagonists strongly suggest that GluN2ANMDARs do not significantly contribute to the responses we observed. However, to our knowledge it is not published or known whether Ro 25-6981 can inhibit triheteromeric receptors, nor whether it does so less effectively than ifenprodil, so the precise proportion of GluN2B and triheteromeric receptor contribution remains unclear. Nevertheless, based on the dramatic reduction in the number of events with $3 \mu \mathrm{m}$ ifenprodil treatment and the consistency across drug treatments, it is likely that GluN2B-NMDARs mediate the majority of the $\mathrm{Ca}^{2+}$ influx because of spontaneous release at these synapses.

We observed a high degree of variability in mSCaT amplitude both between and within synapses. Differences in the number of receptors activated per event or the NMDAR subtype activated could underlie variability in event amplitude. If the number of NMDARs activated per event was dominating this inter-event variability, then this would lead to the prediction that some synapses with more NMDARs, or a higher density of NMDARs apposed to release sites would have overall larger events. One consequence of this would be that the variability within a single synapse would be smaller than the variability between synapses. However, we observed a similar amount of mSCaT amplitude variability within synapses as between synapses, thus difference in the number of NMDARs able to be activated between synapses is not likely the dominant source of variability. GluN2B-NMDARs have longer open times and longer burst duration than
GluN2A-NMDARs but have a much lower open probability, suggesting that they may contribute more to variability in the amount of $\mathrm{Ca}^{2+}$ influx per event (Santucci and Raghavachari, 2008). In fact, in single-channel recordings, ifenprodil reduces variability of NMDAR total open time (Piña-Crespo and Gibb, 2002). Consistent with this prediction, we observed there was a decrease in mean CV of mSCaT amplitude from 0.6 to 0.5 following ifenprodil treatment. This suggests that variability in $\mathrm{Ca}^{2+}$ influx through GluN2B-NMDARs substantially contributes to the differences in mSCaT amplitude between events, and that $\mathrm{Ca}^{2+}$ influx through activated GluN2A-NMDARs is less variable.

Spine size was not substantially correlated with the amount of $\mathrm{Ca}^{2+}$ influx per event. Similarly, NMDAR activation does not scale with spine size following glutamate uncaging (Sobczyk et al., 2005; Takasaki and Sabatini, 2014) or evoked release (Nimchinsky et al., 2004). Together, these observations indicate that the amount of NMDAR activation is independent from spine size for both release modes. Another spine feature that may alter mSCaT amplitude and contribute to variability is spine neck diameter, because this could alter $\mathrm{Ca}^{2+}$ retention in the spine (Svoboda et al., 1996) Additionally, although we did not measure a strong correlation between spine size and mSCaT amplitude, it is nevertheless possible that small fluctuations in spine area during the course of the experiment could contribute slightly to the variability observed in mSCaT amplitude. Furthermore, PSD size measured with correlative super-resolution imaging was also unrelated to NMDAR activation. We did observe a weak negative correlation between spine size and $\mathrm{mSCaT}$ amplitude, however, we suspect that this is because of a decrease not in actual $\mathrm{Ca}^{2+}$ influx, but in the ratio of $\mathrm{Ca}^{2+}$ influx to total GCaMP6f in the compartment (that is, very large spines have a large basal $F$ ).

It is worth highlighting this novel combination of superresolution imaging with functional measures at individual synapses. Nanoscale protein organization is hypothesized to control many aspects of synaptic function and signaling (Bourne and Harris, 2012; Choquet and Triller, 2013; Biederer et al., 2017; Chamma and Thoumine, 2018), so this correlative approach will be important for future tests of how NMDAR activation is impacted by other nanoscale synaptic features, such as the presence of subsynaptic scaffold nanoclusters (Fukata et al., 2013; MacGillavry et al., 2013; Nair et al., 2013; Broadhead et al., 2016; Tang et al., 2016). Rapidly improving and diversifying sensors for neurotransmitters and intracellular messengers (Marvin et al., 2013; Mehta et al., 2018; Ross et al., 2018), also suggest that it will be possible to more directly measure the link between synapse nanostructure and both the ionotropic and non-ionotropic activity of the receptor. Conversely, synaptic function can alter subsynaptic protein distributions, potentially mediating aspects of functional plasticity (Glebov et al., 2017; Chen et al., 2018). We anticipate the correlative approach will be integral for exploration of dynamic synaptic signaling.

In another series of experiments, we asked whether synapse distance from the soma or degree of branch 
complexity plays a role in variability in the amount of NMDAR activation. Previous work has suggested that NMDAR-mediated $\mathrm{Ca}^{2+}$ influx is larger at synapses further away from the soma (Walker et al., 2017). Our data were consistent with this finding and revealed a significant correlation of mSCaT amplitude with the distance of the synapse from the soma, but not with branch complexity. However, there is evidence that spine size is correlated with distance from the soma and that more distal synapses tend to be smaller than those more proximal (Katz et al., 2009; Walker et al., 2017). Therefore, the relationship between spine distance and event amplitude may in part arise simply from a larger proportional $\mathrm{Ca}^{2+}$ influx at a subset of distal spines that are much smaller than proximal spines. Additionally, we found that the amount of blockade with ifenprodil was unrelated to synapse position. Overall, we conclude that despite a slight correlation between synapse position and mSCaT amplitude, synapse position is not a primary modulator of NMDARmediated $\mathrm{Ca}^{2+}$ influx between synapses.

Major remaining options for the source of variability in NMDAR activation are phosphorylation state of NMDARs, presynaptic changes in the amount of glutamate released between events, or finally, random variation in channel open time. Phosphorylation of the NMDAR can modify channel conductance and lead to changes in $\mathrm{Ca}^{2+}$ influx (Wang and Salter, 1994; Salter and Kalia, 2004; Skeberdis et al., 2006; Chen and Roche, 2007). These changes can occur on the timescale of minutes (Wang and Salter, 1994), which although not completely incompatible with our average mSCaT frequency of $\sim 2$ events per minute, is unlikely to have contributed substantially to withinsynapse variance in our experiments. However, it has been shown that preventing receptor phosphorylation alters basal transmission (Wang and Salter, 1994; Skeberdis et al., 2006), which suggests that there is some basal level of NMDAR phosphorylation ongoing that can modify NMDAR properties. Thus, although it is unclear whether NMDAR receptor phosphorylation would be sufficient to mediate the large amount of variability observed within synapses, it appears able to play a role in distinguishing NMDAR-mediated $\mathrm{Ca}^{2+}$ influx between synapses.

Differences in the amount of glutamate release per event have long been thought to be a major contributor to variability in the amount of receptor activation by spontaneous release (Bekkers et al., 1990; Liu et al., 1999; McAllister and Stevens, 2000; Hanse and Gustafsson, 2001; Franks et al., 2003). Modeling the amount of AMPAR activation with varying quantal size has demonstrated an increase in the number of activated receptors as glutamate molecules per vesicle is increased (Franks et al., 2003). Despite the likely small number of NMDARs activated per event (Nimchinsky et al., 2004), it is possible that there is variability in the number of NMDARs activated that is in part because of differences in the amount of glutamate released at each event. In addition to the amount of glutamate per vesicle, the release site position with respect to receptor position could also underlie variability in the amount of receptor activation. Modeling receptor activation due to glutamate release at different distances from the highest density of receptors has suggested that release site position could play a large role in the variability in response amplitude (Uteshev and Pennefather, 1996; MacGillavry et al., 2013; Savtchenko and Rusakov, 2013). Especially in the case of spontaneous release which may occur more randomly across the active zone than evoked release (Tang et al., 2016), release position may play a role in inter-event variability. Finally, another likely source of variability in $\mathrm{Ca}^{2+}$ influx per event is stochastic channel open-close transitions (Franks et al., 2003). GluN2B-NMDARs have a relatively low open probability (Chen et al., 1999), which could produce large essentially random changes in the amount of $\mathrm{Ca}^{2+}$ influx per event (Yeung et al., 2004; Zeng and Holmes, 2010). Especially in this case where there are very few NMDARs activated per event, these random fluctuations could dominate the variability.

Overall, we conclude that the high degree of variability in the amount of $\mathrm{Ca}^{2+}$ influx through spontaneous activated NMDARs is not primarily because of synapsespecific features including the number of available receptors, NMDAR subtype, synapse size, or synapse position within the dendritic tree. Rather, the high degree of variability of spontaneous NMDAR activation is most likely dominated by nanoscale intrinsic properties of the synapse that influence receptor activation probability, including receptor position, release site position, and the glutamate concentration profile following each release event, and by the highly varying open time of the channels that do become activated.

\section{References}

Andreae LC, Ben Fredj N, Burrone J (2012) Independent vesicle pools underlie different modes of release during neuronal development. J Neurosci 32:1867-1874.

Andreae LC, Burrone J (2015) Spontaneous neurotransmitter release shapes dendritic arbors via long-range activation of NMDA receptors. Cell Rep 10:873-882.

Aoto J, Nam Cl, Poon MM, Ting P, Chen L (2008) Synaptic signaling by all-trans retinoic acid in homeostatic synaptic plasticity. Neuron 60:308-320.

Aow J, Dore K, Malinow R (2015) Conformational signaling required for synaptic plasticity by the NMDA receptor complex. Proc Natl Acad Sci U S A 112:14711-14716.

Araki Y, Zeng M, Zhang M, Huganir Richard LL (2015) Rapid dispersion of SynGAP from synaptic spines triggers AMPA receptor insertion and spine enlargement during LTP. Neuron 85:173-189.

Ascher P, Nowak L (1988) The role of divalent cations in the $\mathrm{N}$-methyl-D-aspartate responses of mouse central neurones in culture. J Physiol 399:247-266.

Atasoy D, Ertunc M, Moulder KL, Blackwell J, Chung C, Su J, Kavalali ET (2008) Spontaneous and evoked glutamate release activates two populations of NMDA receptors with limited overlap. J Neurosci 28:10151-10166.

Autry AE, Adachi M, Nosyreva E, Na ES, Los MF, Cheng PF, Kavalali ET, Monteggia LM (2011) NMDA receptor blockade at rest triggers rapid behavioural antidepressant responses. Nature 475:91-96.

Barth AL, Malenka RC (2001) NMDAR EPSC kinetics do not regulate the critical period for LTP at thalamocortical synapses. Nat Neurosci 4:235-236.

Bekkers JM, Stevens CF (1989) NMDA and non-NMDA receptors are co-localized at individual excitatory synapses in cultured rat hippocampus. Nature 341:230-233. 
Bekkers JM, Richerson GB, Stevens CF (1990) Origin of variability in quantal size in cultured hippocampal neurons and hippocampal slices. Proc Natl Acad Sci U S A 87:5359-5362.

Biederer T, Kaeser PS, Blanpied TA (2017) Transcellular nanoalignment of synaptic function. Neuron 96:680-696.

Bourne JN, Harris KM (2012) Nanoscale analysis of structural synaptic plasticity. Curr Opin Neurobiol 22:372-382.

Broadhead MJ, Horrocks MH, Zhu F, Muresan L, BenavidesPiccione R, DeFelipe J, Fricker D, Kopanitsa MV, Duncan RR, Klenerman D, Komiyama NH, Lee SF, Grant SGN (2016) PSD95 nanoclusters are postsynaptic building blocks in hippocampus circuits. Sci Rep 6:24626.

Chamma I, Thoumine O (2018) Dynamics, nanoscale organization, and function of synaptic adhesion molecules. Mol Cell Neurosci 91:95-107.

Chen BS, Roche KW (2007) Regulation of NMDA receptors by phosphorylation. Neuropharmacology 53:362-368.

Chen H, Tang AH, Blanpied TA, Burrone J, Holzbaur E (2018) Subsynaptic spatial organization as a regulator of synaptic strength and plasticity. Curr Opin Neurobiol 51:147-153.

Chen L, Cooper NG, Mower GD (2000) Developmental changes in the expression of NMDA receptor subunits (NR1, NR2A, NR2B) in the cat visual cortex and the effects of dark rearing. Brain Res Mol Brain Res 78:196-200.

Chen N, Luo T, Raymond LA (1999) Subtype-dependence of NMDA receptor channel open probability. J Neurosci 19:6844-6854.

Chen X, Levy JM, Hou A, Winters C, Azzam R, Sousa AA, Leapman RD, Nicoll RA, Reese TS (2015) PSD-95 family MAGUKs are essential for anchoring AMPA and NMDA receptor complexes at the postsynaptic density. Proc Natl Acad Sci U S A 112:E6983E6992.

Choquet D, Triller A (2013) The dynamic synapse. Neuron 80:691703.

Cummings JA, Mulkey RM, Nicoll RA, Malenka RC (1996) $\mathrm{Ca}^{2+}$ signaling requirements for long-term depression in the hippocampus. Neuron 16:825-833.

Dore K, Aow J, Malinow R (2015) Agonist binding to the NMDA receptor drives movement of its cytoplasmic domain without ion flow. Proc Natl Acad Sci U S A 112:14705-14710.

Dore K, Aow J, Malinow R (2016) The emergence of NMDA receptor metabotropic function: Insights from imaging. Front Synaptic Neurosci 8:20.

Dore K, Stein IS, Brock JA, Castillo PE, Zito K, Sjöström PJ (2017) Unconventional NMDA receptor signaling. J Neurosci 37:1080010807.

Dupuis JP, Ladépêche L, Seth H, Bard L, Varela J, Mikasova L, Bouchet D, Rogemond V, Honnorat J, Hanse E, Groc L (2014) Surface dynamics of GluN2B-NMDA receptors controls plasticity of maturing glutamate synapses. EMBO J 33: 842-861.

El-Husseini AED, Schnell E, Chetkovich DM (2000) PSD-95 involvement in maturation of excitatory synapses. Science 290:13641368.

Fischer G, Mutel V, Trube G, Malherbe P, Kew JN, Mohacsi E, Heitz MP, Kemp JA (1997) Ro 25-6981, a highly potent and selective blocker of N-methyl-D-aspartate receptors containing the NR2B subunit. Characterization in vitro. J Pharmacol Exp Ther 283:12851292.

Foster KA, McLaughlin N, Edbauer D, Phillips M, Bolton A, Constantine-Paton M, Sheng M (2010) Distinct roles of NR2A and NR2B cytoplasmic tails in long-term potentiation. J Neurosci 30: $2676-2685$

Frank CA, Kennedy MJ, Goold Carleton PP, Marek KW, Davis GWW (2006) Mechanisms underlying the rapid induction and sustained expression of synaptic homeostasis. Neuron 52:663-677.

Franks KM, Stevens CF, Sejnowski TJ (2003) Independent sources of quantal variability at single glutamatergic synapses. J Neurosci 23:3186-3195.

Fredj NB, Burrone J (2009) A resting pool of vesicles is responsible for spontaneous vesicle fusion at the synapse. Nat Neurosci 12: 751-758.
Fukata Y, Dimitrov A, Boncompain G, Vielemeyer O, Perez F, Fukata M (2013) Local palmitoylation cycles define activity-regulated postsynaptic subdomains. J Cell Biol 202:145-161.

Gambrill AC, Barria A (2011) NMDA receptor subunit composition controls synaptogenesis and synapse stabilization. Proc Natl Acad Sci U S A 108:5855-5860.

Glebov OO, Jackson RE, Winterflood CM, Owen DM, Barker EA, Doherty P, Ewers H, Burrone J (2017) Nanoscale structural plasticity of the active zone matrix modulates presynaptic function. Cell Rep 18:2715-2728.

Gray JA, Shi Y, Usui H, During MJ, Sakimura K, Nicoll RA (2011) Distinct modes of AMPA receptor suppression at developing synapses by GluN2A and GluN2B: single-cell NMDA receptor subunit deletion in vivo. Neuron 71:1085-1101.

Groemer TW, Klingauf J (2007) Synaptic vesicles recycling spontaneously and during activity belong to the same vesicle pool. Nat Neurosci 10:145-147.

Hanse E, Gustafsson B (2001) Quantal variability at glutamatergic synapses in area CA1 of the rat neonatal hippocampus. J Physiol 531:467-480.

Hansen KB, Ogden KK, Yuan H, Traynelis SF (2014) Distinct functional and pharmacological properties of Triheteromeric GluN1/ GluN2A/GluN2B NMDA receptors. Neuron 81:1084-1096.

Hardingham GE, Arnold FJ, Bading H (2001) A calcium microdomain near NMDA receptors: on switch for ERK-dependent synapse-tonucleus communication. Nat Neurosci 4:565-566.

Hardingham GE, Bading $\mathrm{H}$ (2003) The yin and yang of NMDA receptor signalling. Trends Neurosci 26:81-89.

Harris KM, Stevens JK (1989) Dendritic spines of CA 1 pyramidal cells in the rat hippocampus: serial electron microscopy with reference to their biophysical characteristics. J Neurosci 9:29822997.

Harris KM, Weinberg RJ, Hill C, Carolina N (2014) Ultrastructure of synapses in the mammalian brain. Cold Spring Harb Perspect Biol 4:a005587.

Hatton CJ, Paoletti P (2005) Modulation of triheteromeric NMDA receptors by N-terminal domain ligands. Neuron 46:261-274.

Higley MJ, Sabatini BL (2012) Calcium signaling in dendritic spines. Cold Spring Harb Perspect Biol 4:a005686.

Hua Y, Sinha R, Martineau M, Kahms M, Klingauf J (2010) A common origin of synaptic vesicles undergoing evoked and spontaneous fusion. Nat Neurosci 13:1451-1453.

Huganir RL, Nicoll RA (2013) AMPARs and synaptic plasticity: the last 25 years. Neuron 80:704-717.

Inoue A, Okabe S (2003) The dynamic organization of postsynaptic proteins: translocating molecules regulate synaptic function. Curr Opin Neurobiol 13:332-340.

Kannangara TS, Bostrom CA, Ratzlaff A, Thompson L, Cater RM, Gil-Mohapel J, Christie BR (2014) Deletion of the NMDA receptor GluN2A subunit significantly decreases dendritic growth in maturing dentate granule neurons. PLoS One 9:e103155.

Katz Y, Menon V, Nicholson DA, Geinisman Y, Kath WL, Spruston N (2009) Synapse distribution suggests a two-stage model of dendritic integration in CA1 pyramidal neurons. Neuron 63:171-177.

Kavalali ET, Chung C, Khvotchev M, Leitz J, Nosyreva E, Raingo J, Ramirez DMO (2011) Spontaneous neurotransmission: an independent pathway for neuronal signaling? Physiology 26:45-53.

Kellermayer B, Ferreira JS, Dupuis J, Levet F, Grillo-Bosch D, Bard L, Linares-Loyez J, Bouchet D, Choquet D, Rusakov DA, Bon P, Sibarita JB, Cognet L, Sainlos M, Carvalho AL, Groc L (2018) Differential nanoscale topography and functional role of GluN2NMDA receptor subtypes at glutamatergic synapses. Neuron 100: 106-119.e7.

Kennedy MB (2000) Signal-processing machines at the postsynaptic density. Science 290:750-754.

Kessels HW, Nabavi S, Malinow R (2013) Metabotropic NMDA receptor function is required for $\beta$-amyloid-induced synaptic depression. Proc Natl Acad Sci U S A 110:4033-4038. 
Kharazia VN, Weinberg RJ (1999) Immunogold Localization of AMPA and NMDA receptors in somatic sensory cortex of albino rat. $J$ Comp Neurol 412:292-302.

Kuner T, Schoepfer R (1996) Multiple structural elements determine subunit specificity of $\mathrm{Mg}^{2+}$ block in NMDA receptor channels. $J$ Neurosci 16:3549-3558.

Levy AD, Xiao X, Shaw JE, Sudarsana Devi SP, Katrancha SM, Bennett AM, Greer CA, Howe JR, Machida K, Koleske AJ (2018) Noonan syndrome-associated SHP2 dephosphorylates GluN2B to regulate NMDA receptor function. Cell Rep 24:1523-1535.

Liu G, Choi S, Tsien RW (1999) Variability of neurotransmitter concentration and nonsaturation of postsynaptic AMPA receptors at synapses in hippocampal cultures and slices. Neuron 22:395-409.

MacDermott AB, Mayer ML, Westbrook GL, Smith SJ, Barker JL (1986) NMDA-receptor activation increases cytoplasmic calcium concentration in cultured spinal cord neurones. Nature 321:519522.

MacGillavry HD, Song Y, Raghavachari S, Blanpied TA (2013) Nanoscale scaffolding domains within the postsynaptic density concentrate synaptic AMPA receptors. Neuron 78:615-622.

Malenka RC, Bear MF (2004) LTP and LTD: an embarrassment of riches. Neuron 44:5-21.

Marvin JS, Borghuis BG, Tian L, Cichon J, Harnett MT, Akerboom J, Gordus A, Renninger SL, Chen TW, Bargmann Cl, Orger MB, Schreiter ER, Demb JB, Gan WB, Hires SA, Looger LL (2013) An optimized fluorescent probe for visualizing glutamate neurotransmission. Nat Methods 10:162-170.

Matsuzaki M, Ellis-Davies GC, Nemoto T, Miyashita Y, lino M, Kasai H (2001) Dendritic spine geometry is critical for AMPA receptor expression in hippocampal CA1 pyramidal neurons. Nat Neurosci 4:1086-1092.

Matsuzaki M, Honkura N, Ellis-Davies GCR, Kasai H (2004) Structural basis of long-term potentiation in single dendritic spines. Nature 429:761-766.

McAllister AK, Stevens CF (2000) Nonsaturation of AMPA and NMDA receptors at hippocampal synapses. Proc Natl Acad Sci U S A 97:6173-6178.

Mehta S, Zhang Y, Roth RH, Zhang JF, Mo A, Tenner B, Huganir RL, Zhang J (2018) Single-fluorophore biosensors for sensitive and multiplexed detection of signalling activities. Nat Cell Biol 20: 1215-1225.

Nabavi S, Kessels HW, Alfonso S, Aow J, Fox R, Malinow R (2013) Metabotropic NMDA receptor function is required for NMDA receptor-dependent long-term depression. Proc Natl Acad Sci U S A 110:4027-4032.

Nair D, Hosy E, Petersen JD, Constals A, Giannone G, Choquet D, Sibarita JB (2013) Super-resolution imaging reveals that AMPA receptors inside synapses are dynamically organized in nanodomains regulated by PSD95. J Neurosci 33:13204-13224.

Nimchinsky EA, Yasuda R, Oertner TG, Svoboda K (2004) The number of glutamate receptors opened by synaptic stimulation in single hippocampal spines. J Neurosci 24:2054-2064.

Paoletti P (2011) Molecular basis of NMDA receptor functional diversity. Eur J Neurosci 33:1351-1365.

Paoletti P, Bellone C, Zhou Q (2013) NMDA receptor subunit diversity: impact on receptor properties, synaptic plasticity and disease. Nat Rev Neurosci 14:383-400.

Piña-Crespo JC, Gibb AJ (2002) Subtypes of NMDA receptors in new-born rat hippocampal granule cells. J Physiol 541:41-64.

Prange O, Murphy TH (1999) Correlation of miniature synaptic activity and evoked release probability in cultures of cortical neurons. $J$ Neurosci 19:6427-6438.

Prybylowski K, Wenthold RJ (2004) N-methyl-D-aspartate receptors: subunit assembly and trafficking to the synapse. J Biol Chem 279:9673-9676.

Prybylowski K, Chang K, Sans N, Kan L, Vicini S, Wenthold RJ (2005) The synaptic localization of NR2B-containing NMDA receptors is controlled by interactions with PDZ proteins and AP-2. Neuron 47:845-857.
Ramirez DMO, Kavalali ET (2011) Differential regulation of spontaneous and evoked neurotransmitter release at central synapses. Curr Opin Neurobiol 21:275-282.

Rauner C, Köhr G (2011) Triheteromeric NR1/NR2A/NR2B receptors constitute the major $N$-methyl-D-aspartate receptor population in adult hippocampal synapses. J Biol Chem 286:7558-7566.

Reese AL, Kavalali ET (2015) Spontaneous neurotransmission signals through store-driven $\mathrm{Ca}^{+}$transients to maintain synaptic homeostasis. eLife 4:e09262.

Reese AL, Kavalali ET (2016) Single synapse evaluation of the postsynaptic NMDA receptors targeted by evoked and spontaneous neurotransmission. eLife 5:e21170.

Ritter LM, Vazquez DM, Meador-Woodruff JH (2002) Ontogeny of ionotropic glutamate receptor subunit expression in the rat hippocampus. Brain Res Dev Brain Res 139:227-236.

Ross BL, Tenner B, Markwardt ML, Zviman A, Shi G, Kerr JP, Snell NE, McFarland JJ, Mauban JR, Ward CW, Rizzo MA, Zhang J (2018) Single-color, ratiometric biosensors for detecting signaling activities in live cells. eLife 7:e35458

Salter MW, Kalia LV (2004) Src kinases: a hub for NMDA receptor regulation. Nat Rev Neurosci 5:317-328.

Sanhueza M, Fernandez-Villalobos G, Stein IS, Kasumova G, Zhang P, Bayer KU, Otmakhov N, Hell JW, Lisman J (2011) Role of the CaMKII/NMDA receptor complex in the maintenance of synaptic strength. J Neurosci 31:9170-9178.

Santucci DM, Raghavachari S (2008) The effects of NR2 subunitdependent NMDA receptor kinetics on synaptic transmission and CaMKII activation. PLoS Comput Biol 4:e1000208.

Sara Y, Virmani T, Deák F, Liu X, Kavalali ET (2005) An isolated pool of vesicles recycles at rest and drives spontaneous neurotransmission. Neuron 45:563-573.

Sara Y, Bal M, Adachi M, Monteggia LM, Kavalali ET (2011) Usedependent AMPA receptor block reveals segregation of spontaneous and evoked glutamatergic neurotransmission. 31:53785382.

Savtchenko LP, Rusakov DA (2013) Moderate AMPA receptor clustering on the nanoscale can efficiently potentiate synaptic current. Philos Trans R Soc Lond B Biol Sci 369:20130167.

Schikorski T, Stevens CF (1997) Quantitative ultrastructural analysis of hippocampal excitatory synapses. J Neurosci 17:5858-5867.

Shinohara Y, Hirase H, Watanabe M, Itakura M, Takahashi M, Shigemoto R (2008) Left-right asymmetry of the hippocampal synapses with differential subunit allocation of glutamate receptors. Proc Natl Acad Sci U S A 105:19498-19503.

Shipton OA, Paulsen O (2014) GluN2A and GluN2B subunitcontaining NMDA receptors in hippocampal plasticity. Philos Trans R Soc Lond B Biol Sci 369:20130163.

Sinnen BL, Bowen AB, Gibson ES, Kennedy MJ (2016) Local and use-dependent effects of -amyloid oligomers on NMDA receptor function revealed by optical quantal analysis. J Neurosci 36: 11532-11543.

Skeberdis VA, Chevaleyre V, Lau CG, Goldberg JH, Pettit DL, Suadicani SO, Lin Y, Bennett MV, Yuste R, Castillo PE, Zukin RS (2006) Protein kinase $A$ regulates calcium permeability of NMDA receptors. Nat Neurosci 9:501-510.

Sobczyk A, Scheuss V, Svoboda K (2005) NMDA receptor subunitdependent $[\mathrm{Ca} 2+]$ signaling in individual hippocampal dendritic spines. J Neurosci 25:6037-6046.

Stein IS, Gray JA, Zito K (2015) Non-ionotropic NMDA receptor signaling drives activity-induced dendritic spine shrinkage. J Neurosci 35:12303-12308.

Stroebel D, Carvalho S, Grand T, Zhu S, Paoletti P (2014) Controlling NMDA receptor subunit composition using ectopic retention signals. J Neurosci 34:16630-16636.

Stroebel D, Casado M, Paoletti P (2018) Triheteromeric NMDA receptors: from structure to synaptic physiology. Curr Opin Physiol 2:1-12.

Sutton MA, Wall NR, Aakalu GN, Schuman EM (2004) Regulation of dendritic protein synthesis by miniature synaptic events. Science 304:1979-1983. 
Sutton MA, Ito HT, Cressy P, Kempf C, Woo JC, Schuman EM (2006) Miniature neurotransmission stabilizes synaptic function via tonic suppression of local dendritic protein synthesis. Cell 125:785-799.

Sutton MA, Taylor AM, Ito HT, Pham A, Schuman EM (2007) Postsynaptic decoding of neural activity: eEF2 as a biochemical sensor coupling miniature synaptic transmission to local protein synthesis. Neuron 55:648-661.

Svoboda K, Tank DW, Denk W (1996) Direct measurement of coupling between dendritic spines and shafts. Science 272:716-719.

Takasaki K, Sabatini BL (2014) Super-resolution 2-photon microscopy reveals that the morphology of each dendritic spine correlates with diffusive but not synaptic properties. Front Neuroanat 8:29.

Takumi Y, Ramírez-León V, Laake P, Rinvik E, Ottersen OP (1999) Different modes of expression of AMPA and NMDA receptors in hippocampal synapses. Nat Neurosci 2:618-24.

Tang A-H, Chen H, Li TP, Metzbower SR, MacGillavry HD, Blanpied TA (2016) A trans-synaptic nanocolumn aligns neurotransmitter release to receptors. Nature 536:210-214.

Tovar KR, McGinley MJ, Westbrook GL (2013) Triheteromeric NMDA receptors at hippocampal synapses. J Neurosci 33:9150-9160.

Townsend M, Yoshii A, Mishina M, Constantine-Paton M (2003) Developmental loss of miniature $N$-methyl-D-aspartate receptor currents in NR2A knockout mice. Proc Natl Acad Sci U S A 100:1340-1345.

Uteshev VV, Pennefather PS (1996) A mathematical description of miniature postsynaptic current generation at central nervous system synapses. Biophys J 71:1256-1266.

Walker AS, Neves G, Grillo F, Jackson RE, Rigby M, Donnell CO, Lowe AS, Vizcay-Barrena G, Fleck RA, Burrone J (2017) Distance- dependent gradient in NMDAR-driven spine calcium signals along tapering dendrites. Proc Natl Acad Sci U S A 114:E1986-E1995.

Wang YT, Salter MW (1994) Regulation of NMDA receptors by tyrosine kinases and phosphatases. Nature 369:233-235.

Watt AJ, Sjöström PJ, Häusser M, Nelson SB, Turrigiano GG (2004) A proportional but slower NMDA potentiation follows AMPA potentiation in LTP. Nat Neurosci 7:518-524.

Wilhelm BG, Groemer TW, Rizzoli SO (2010) The same synaptic vesicles drive active and spontaneous release. Nat Neurosci 13: 1454-1456.

Wong JM, Gray JA (2018) Long-term depression is independent of GluN2 subunit composition. J Neurosci 38:4462-4470.

Xiao X, Levy AD, Rosenberg BJ, Higley MJ, Koleske AJ (2016) Disruption of coordinated presynaptic and postsynaptic maturation underlies the defects in hippocampal synapse stability and plasticity in Abl2/Arg-deficient mice. J Neurosci 36:6778-6791.

Yashiro K, Philpot BD (2008) Regulation of NMDA receptor subunit expression and its implications for LTD, LTP, and metaplasticity. Neuropharmacology 55:1081-1094.

Yeung LC, Castellani GC, Shouval HZ (2004) Analysis of the intraspinal calcium dynamics and its implications for the plasticity of spiking neurons. Phys Rev E Stat Nonlin Soft Matter Phys 69: 011907.

Zeng S, Holmes WR (2010) The effect of noise on CaMKII activation in a dendritic spine during LTP induction. J Neurophysiol 103: 1798-1808.

Zhao JP, Constantine-Paton M (2007) NR2A/Mice lack long-term potentiation but retain NMDA receptor and L-Type $\mathrm{Ca}^{2+}$ Channeldependent long-term depression in the juvenile superior colliculus. J Neurosci 27:13649-13654. 